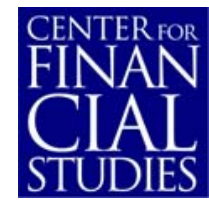

No. $2006 / 29$

Public Policy and Venture Capital Financed Innovation: A Contract Design Approach

Julia Hirsch 


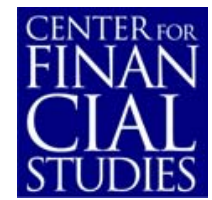

\section{Center for Financial Studies}

The Center for Financial Studies is a nonprofit research organization, supported by an association of more than 120 banks, insurance companies, industrial corporations and public institutions. Established in 1968 and closely affiliated with the University of Frankfurt, it provides a strong link between the financial community and academia.

The CFS Working Paper Series presents the result of scientific research on selected topics in the field of money, banking and finance. The authors were either participants in the Center's Research Fellow Program or members of one of the Center's Research Projects.

If you would like to know more about the Center for Financial Studies, please let us know of your interest.

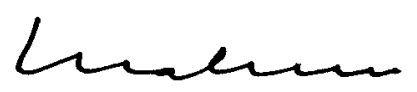

Prof. Dr. Jan Pieter Krahnen

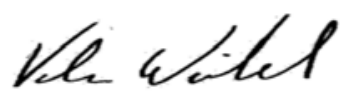

Prof. Volker Wieland, Ph.D. 


\title{
Public Policy and Venture Capital Financed Innovation: A Contract Design Approach*
}

\author{
Julia Hirsch ${ }^{1}$ \\ This Version \\ December 2006
}

\begin{abstract}
:
The effects of public policy programs which aim at internalizing spill-overs due to successful innovation are analyzed in a sequential double-sided moral hazard doublesided adverse selection framework. The central focus lies in analyzing their impact on contract design. We show that in our framework only ex post grants are a robust instrument for implementing the first-best situation, whereas the success of guarantee programs, ex ante grants and some types of investment grants depends strongly on the characteristics of the project: in certain cases they not only give no further incentives but even destroy contract mechanisms and so worsen the outcome.
\end{abstract}

JEL Classification: D82, G24, G32, H25, H81

Keywords: Public Policy, Contract Design, Venture Capital, Moral Hazard, Asymmetric Information

\footnotetext{
* This paper forms the third chapter of my PhD thesis at Goethe University Frankfurt. I thank Christian Keuschnigg, Ibolya Schindele, Uwe Walz, the participants at the annual meeting of the Verein für Socialpolitik 2005, the participants at the Second RICAFE Conference on Risk Capital and the Financing of European Innovative Firms, the participants at the CPE-CERESS Workshop on Public Policy and StartUp Firms and seminar participants at Goethe Universität Frankfurt for valuable comments and suggestions. All errors of course remain my own. Financial support from the European Commission, grant HPSE-CT-2002-00140, is gratefully acknowledged. 


\section{Introduction}

Policies to foster innovation are widespread, as without innovation an economy would stagnate, with obvious negative effects on the welfare of future generations. Financial development is particularly important against the background of financing constraints for young innovative firms with few tangible assets (see e.g. Audretsch and Thurik [2001], Rajan and Zingales [2001]); the venture capital industry in particular plays a crucial role in the financing of these firms (see Kortum and Lerner [2000]). Thus it is not surprising that a broad range of public policy programs aims at fostering innovation by supporting the venture capital industry. The European Investment Fund's venture capital portfolio, for example, amounts to more than 2.5 billion euros invested in more than 185 venture capital funds. Thus the EIF has indirectly supported more than 1800 high-tech firms in Europe (see EIB [2004]).

But the crucial question is - provided that governments should intervene in the venture capital industry - how public policy programs should be optimally designed. This paper evaluates different public policy measures in order to fill the gap in research on public policy in the venture capital area - a particularly crucial gap, given the importance of the venture capital industry and the high intensity of public support. To be more precise, the present paper will study in detail if and how the optimal contract design between the venture capitalists and their portfolio firms is influenced by public intervention, whether this modification has any impact on the incentives and thus on the behavior of the contracting parties, and finally, it will evaluate its impact on the market outcome as a whole.

To the best of our knowledge, this paper is the first to analyze public policy measures by focussing on their impact on contract design. We think, however, that, due to the prevailing role of explicit contracts in the venture capital industry, public policy measures 
can only be evaluated correctly when taking this impact into account. Explicit contracts play such a predominant role because they are necessary for the solution of the severe moral hazard and adverse selection problems resulting from the specific characteristics of this industry, namely the active involvement of venture capitalists in the operations of the start-up firms and the high degree of uncertainty to which venture capitalists are exposed regarding the value of their investments, exacerbated by the small collateral platform from which entrepreneurs operate. Moreover, as the partnership between entrepreneur and venture capitalist is of limited duration (the venture capitalist's objective is to exit, i.e. to make a return by getting out), it is not possible to solve these conflicts of interest implicitly. In fact, empirical studies confirm the existence of complex and highly sensitive contracts which not only determine the cash-flow rights of the contracting parties but also the specific control and decision rights of the venture capitalist (e.g. Kaplan and Strömberg [2003]).

In order to allow for public intervention, we address potential inefficiencies in the innovation process. We model the mentioned double-sided adverse selection and doublesided moral hazard problems and, additionally, introduce a spill-over effect on the rest of the economy which is related to the realization of innovative projects. Thus within our framework, the market solution will never be efficient: while the contracting parties may be able to solve the asymmetric information problems, they will never take the spill-over effect into account. But this spill-over effect can be motivated by both empirical insights and theoretical foundations. We take the OECD [2001] study as a starting point: "Although venture capital does not aim at supporting R\&D per se, its substantial emphasis on small, high-technology businesses has enabled markets to become considerably more capable of sustaining large, risky investments in R\&D in early business stages (...) The influx of venture capital does not necessarily remove the rationale for government support for SMEs 
because significant discrepancies can still exist between private and social returns to $R \& D$ and innovation, even in sectors that receive considerable private capital. Recent research indicates that the most successful government-funded small-business projects have been in industry sectors that boast high levels of private venture capital. This finding suggests that private venture capital signals the presence of significant technological findings in a field, and that government funding can stimulate additional exploitation of those opportunities." Indeed, the existence of the described spill-over effect is supported by empirical as well as theoretical evidence: Griliches [1992] shows that highly innovative projects - such as those financed by venture capital - involve a spill-over effect on the rest of the economy. Their social rate of return can be twice as high as their corresponding private rate of return. And the literature on endogenous growth distinguishes three effects of R\&D: the positive effect of innovations on the profitability of other firms as shown by Romer [1986], the so-called consumer-surplus effect, and the business-stealing effect - the extraction of rents of one firm by another - as modelled by Aghion and Howitt [1992]. If the first two effects are sufficiently high - as it normally is for highly innovative projects - the spill-over effect on the rest of the economy will be positive.

Finally, in order to evaluate the existing public policy measures in the venture capital industry we have identified their main mechanisms and accordingly constructed five stylized program categories for analysis: ex post grants, guarantee programs, ex ante grants, investment grants and public support. Ex post grants can be interpreted as tax breaks and there is a great variety of programs which can be classified as ex ante grants. The awards of the "Small Business Innovation Research Program" or those of the "Advanced Technology Program" in the United States are among the most prominent examples. Guarantees have also been an important ingredient of programs in many countries: in Germany, for example, risk transfer was an essential component of many programs of KfW until 2004 
and a guarantee program targeted at investments in later stage firms still exists; in the Netherlands, the "Private Participation Guarantee Order Scheme" was in operation between 1981 and 1995; the Banque du Développement des Petites et Moyennes Entreprises in France and the Austria Wirtschaftsservice Gesellschaft both give specific guarantees. Direct supply of capital by the government in order to increase the investment of the venture capitalists (we classify this form in the investment grants category) is organized in different ways, as can be observed in various current and former programs of KfW in Germany, of the European Investment Fund, or of the "Small Business Investment Companies Program" in the United States. Finally, public support is not very common but the European Investment Fund, for example, has the "Seed Capital Action Program" whereby part of the VC's management costs are covered. We will show that within our framework only ex post grants can implement the first-best situation independently of specification issues. The success of guarantee programs, ex ante grants and some types of investment grants, on the contrary, depends strongly on project characteristics as well as specification issues: sometimes they not only fail to provide further incentives to the contracting parties but even destroy contract mechanisms and so worsen the outcome.

Our paper is related to two strains of literature: first, the papers which deal with public policy in the venture capital area and second, the broad literature on optimal contract design between entrepreneurs and venture capitalists (see Tykvova [2000] for an overview). As regards the first strain of literature, only very few important papers exist. In an empirical study, Lerner [1999] examines the "Small Business Innovation Research Program" in the United States and finds that firms which had received awards were more likely to attract venture capital finance. In a second article, Lerner [2002] reviews the rationale for public policy programs and comments on their design. Da Rin et al. [2004] examine how public policy can contribute to increasing the share of venture capital 
investments in innovative companies, finding that the availability of exit channels plays an important role and that a reduction of the capital gains tax as well as a reduction of labor regulation yield such an increase. The only theoretical contributions to deal with public policy in a venture capital framework with a moral hazard problem are, to our knowledge, the papers by Keuschnigg and Keuschnigg and Nielsen (see, for example, Keuschnigg [2003], Keuschnigg and Nielsen [2003] or Keuschnigg and Nielsen [2004]). They analyze public policy strategies in an equilibrium model but do not model contract design in detail. Thus, as mentioned, our paper is the first to model explicitly the impact of public intervention on contract design.

Therefore, the present study is also related to the broad literature on optimal contract design where we can distinguish two types of papers: those which determine the optimal contract design in a double-sided moral hazard framework and those which analyze the optimal contract design in an adverse selection framework. We think that it is important to combine these two types to do justice to the complexity of reality and so we construct our basic approach in two steps: first, we determine the optimal contract design in a double-sided moral hazard framework; then, in a second step, we introduce a doublesided adverse selection problem. More importantly, this procedure allows us to represent different development stages of the venture capital industry. Our approach is thereby based on the models of Schmidt [2003] and Houben [2002] which provide a good starting point for our analysis as they show that both problems can be solved by adequately designed contracts. Moreover, the mechanisms of these contracts are empirically well supported (see Kaplan and Strömberg [2003]). We modify these models so as to address the public policy issues in a tractable manner.

Other theoretical papers on optimal contract design which are close to the framework of the present paper are Casamatta [2003], Repullo and Suarez [2004] and D'Souza [2001]. 
Casamatta [2003] determines the optimal security design in a double-sided moral hazard framework too, but she shows that convertible securities are only optimal with high investments and that the first-best outcome is not reached. Repullo and Suarez [2004], on the other hand, focus on how a double-sided moral hazard problem in the expansion stage influences the security choice at the beginning of the relationship between entrepreneur and venture capitalist. They show that only when no objective performance indicators pertain after the start-up phase does the initial claim of the venture capitalist correspond to a combination of standard non-linear claims which may be interpreted as convertible security. D'Souza [2001], in contrast, shows the optimality of convertible securities in a framework within which only the venture capitalist has to expend effort and the entrepreneur gets private information about the state of the project after contracting.

This paper proceeds as follows: after presenting our framework which is based on the models of Schmidt [2003] concerning inexperienced and Houben [2002] concerning experienced venture capital markets, we will first focus on inexperienced markets in section 3 analyzing the market outcome without intervention and determining the impact of the different public policy categories afterwards. In a second step, in section 4, we will study how maturation of the market changes the impact of the public policy measures derived. Section 5 summarizes our results. The sixth section makes some robustness checks. Section seven draws together the threads.

\section{The Model}

We consider a market of many venture capitalists (VCs) looking for profitable investment opportunities and some entrepreneurs (Es) with innovative ideas but without financial resources. Therefore, each $\mathrm{E}$ has to convince a $\mathrm{VC}$ to invest an amount of $I$ in his project. 
As the market of VCs is competitive, Es have all the bargaining power ex ante. Moreover, we assume that the VCs and the Es are risk-neutral.

Projects differ only in their inherent innovative value $\alpha$ which is always observable by the contracting parties. Each project can be in three possible states (bad, medium, good) which result from a combination of the market conditions for the innovation and the quality of the E's idea. The market conditions indicate whether the sales expectancy for the product is good or bad and the quality of the idea refers to the technological quality, i.e. the degree of feasibility. The project is of high quality with probability $p$ or of low quality with probability $(1-p)$; and the market conditions for the innovation are either good with probability $q$ or bad with probability $(1-q)$.

If the market conditions are bad and the quality is low, each project will fail and yield a liquidation value of $l(a)$. The liquidation value depends on the effort exerted by the $\mathrm{VC}$ in order to sell off the assets of the company and $l(a)$ is increasing and concave in $a$. Moreover, we assume that the first-best liquidation value $l\left(a_{b}^{*}\right)$ is smaller than the initial investment $I$.

If the market conditions are bad and the quality is high, or if the market conditions are good but the quality is low - we refer to these cases as the medium state of the project - the project is a so-called "living-dead". This means that while the project may leave a return in the amount of the initial investment $I$ it will never generate a higher cash flow - even if both parties exerted high effort levels.

If the market conditions are good and the quality of the idea is high, the project is in the good state and yields a gross surplus of $\bar{x}$, which depends on three factors: the inherent innovative value of the project $(\alpha)$, the effort spent by the $\mathrm{VC}(a)$ and the effort exerted by the $\mathrm{E}(e)$. We interpret the effort of the $\mathrm{E}$ as effort invested in the technological development of the project and that of the $\mathrm{VC}$ as involving managerial contributions. We 
assume that efforts are imperfect substitutes in the good state: if at least one contracting party does not exert any effort, the cash flow amounts only to the initial investment $I$ irrespective of the inherent innovative value:

$$
\bar{x}(0, a, \alpha)=\bar{x}(e, 0, \alpha)=\bar{x}(0,0, \alpha)=I
$$

Otherwise, the cash flow function is increasing and concave in both effort levels and in the inherent innovative value. Effort levels are not complementary at the margin and their marginal impact does not depend on the innovative value $\alpha$ of the project. This is stated formally in the following assumptions.

$$
\begin{aligned}
& \frac{\partial \bar{x}(e, a, \alpha)}{\partial a}>0 \text { and } \frac{\partial^{2} \bar{x}(e, a, \alpha)}{\partial a^{2}}<0 \\
& \frac{\partial \bar{x}(e, a, \alpha)}{\partial e}>0 \text { and } \frac{\partial^{2} \bar{x}(e, a, \alpha)}{\partial e^{2}}<0 \\
& \frac{\partial \bar{x}(e, a, \alpha)}{\partial \alpha}>0 \text { and } \frac{\partial^{2} \bar{x}(e, a, \alpha)}{\partial \alpha^{2}}<0 \\
& \frac{\partial^{2} \bar{x}(e, a, \alpha)}{\partial a \partial e}=0 \\
& \frac{\partial^{2} \bar{x}(e, a, \alpha)}{\partial a \partial \alpha}=0 \quad \text { and } \quad \frac{\partial^{2} \bar{x}(e, a, \alpha)}{\partial e \partial \alpha}=0
\end{aligned}
$$

Thus to sum up, the cash-flows generated by the project $V$ are defined as

$$
\begin{aligned}
V(e, a, \alpha, \theta) & =\bar{x}(e, a, \alpha) & & \text { for } \theta=\operatorname{good} \\
& =I & & \text { for } \theta=\text { medium } \\
& =l(a) & & \text { for } \theta=\operatorname{bad}
\end{aligned}
$$

Furthermore, we assume that the effort of the E and the VC, which is observable but not verifiable, occasions costs to the amount of:

$$
\begin{aligned}
c(e) & =\frac{1}{2} \beta e^{2} \\
c(a) & =\frac{1}{2} \gamma a^{2}
\end{aligned}
$$


In addition, the project yields non-transferable private benefits $B$ to the $\mathrm{E}$ if and only if he has the control rights over the company in at least one period ${ }^{1}$.

Finally, we assume that a project in the good state, whose cash flow exceeds the initial investment costs, induces a spill-over effect on the rest of the economy which is more pronounced for more innovative projects and the effort choices exert an influence on it. The spill-over effect is thus given by

$$
\begin{aligned}
S(e, a, \alpha) & =\varepsilon V(e, a, \alpha) & & \text { for } V(e, a, \alpha)>I \\
& =0 & & \text { otherwise }
\end{aligned}
$$

\begin{tabular}{|c|c|c|c|c|c|c|c|}
\hline$t=0$ & $t=0,5$ & $t=1$ & $t=1,5$ & $t=2$ & $t=3$ & $t=4$ & $t=5$ \\
\hline $\begin{array}{l}\text { Government } \\
\text { announces } \\
\text { public policy } \\
\text { program } \\
\text { (announcement } \\
\text { is binding) }\end{array}$ & $\begin{array}{c}{[\text { Signals] }} \\
\lambda \text { and } \eta]^{* *}\end{array}$ & $\begin{array}{c}\text { Take-it-or- } \\
\text { leave-it-offer } \\
\text { of contract(s) } \\
\text { by E; } \\
\text { Investment } \\
\text { of I by VC }\end{array}$ & $\begin{array}{l}\text { [E and VC } \\
\text { learn the } \\
\text { state of the } \\
\text { project] }{ }^{*}\end{array}$ & $\begin{array}{l}\text { Choice of } \\
\text { effort level e } \\
\text { by E }\end{array}$ & $\begin{array}{c}\text { Choice of } \\
\text { effort level a } \\
\text { by VC }\end{array}$ & $\begin{array}{l}\text { Cash-flow } \\
\text { is realized }\end{array}$ & $\begin{array}{l}\text { Execution } \\
\text { of the } \\
\text { contract }\end{array}$ \\
\hline
\end{tabular}

The time structure of the model is as follows:

Figure 1: Model Timeline

$$
* \text { inexperienced markets } \quad * *=\text { experienced markets }
$$

At $t_{0}$, the government announces its public policy measure. This announcement is costless but binding. The time of execution of the public policy measure depends on the type of measure. At $t_{1}$, the E makes a take-it-or-leave-it offer to the VC. If the VC accepts, $I$ is invested. At $t_{2}$, the $\mathrm{E}$ chooses his ${ }^{2}$ effort level $e$ and at $t_{3}$, the $\mathrm{VC}$ chooses her effort level $a^{3}$. At $t_{4}$, the cash flow of the project is realized and at $t_{5}$, the contract is executed.

\footnotetext{
${ }^{1}$ We assume that $0<B<\min \left[p q \frac{1}{2} \beta e_{g}^{*} 2 ;-L\left(a_{b}^{*}\right)\right]$. This assumption has two implications. First, bad state projects are not socially profitable as $L\left(a_{b}^{*}\right)+B<I$. Second, all privately profitable projects manage to be financed in a private first-best situation despite the limited liability of the $\mathrm{E}$ as $B \leq p q \frac{1}{2} \beta e_{g}^{* 2}$. For details see Appendix A1.

${ }^{2}$ For the reader's convenience in following my argumentation, I refer to the E as he/him and to the $\mathrm{VC}$ as she/her.

${ }^{3}$ See subsection 6 for a comment on the impact of simultaneous effort choice.
} 
As far as the distribution of information is concerned, we analyze two different scenarios in order to be able to focus first on the impact of the double-sided moral hazard problem and the ex ante uncertainty and then on the impact of the double-sided adverse selection problem. However, as mentioned before, even more decisive is the fact that these scenarios can be interpreted as different development stages of the venture capital market, because only in an experienced market are the contracting parties able to acquire private information ex ante. This distinction is crucial as the need for public intervention differs between experienced and inexperienced markets. In fact, we can frequently observe public policy measures which are especially designed for immature markets. Then, however, it is important to know whether these measures have to be absolutely cancelled when reaching a certain development stage or whether they are not harmful in experienced markets. Against this background, we define a first scenario where we assume that no information about the state of the project exists before contracting time (this can be interpreted as the case of an inexperienced market), but that both contracting parties learn the state of the project after the initial investment has sunk (see *). In a second step, we assume that information does exist about the state of the project - thus we are confronted with an experienced market - but we introduce a double-sided asymmetric information problem by assuming that the $\mathrm{E}$ and the $\mathrm{VC}$ each receive a private signal (see ${ }^{* *}$ ). As the E developed the innovative idea, we assume that he receives a perfect signal about the technological quality of the project ( $\bar{\lambda}$ for a high quality and $\underline{\lambda}$ for a low quality project). On the other hand, the VC has gained experience in the venture capital market and thus she has private information about the perspectives of the product market, i.e. she receives a perfect signal about the market conditions for the innovation $(\bar{\eta}$ for good and $\eta$ for bad market conditions). 


\section{The Case of Inexperienced Venture Capital Markets}

Below we will analyze the case of inexperienced markets where no information either about the technological quality of the project or about the market conditions before contracting exists. However, both contracting parties, the $\mathrm{E}$ and the $\mathrm{VC}$, will learn the state of the project before exerting their effort levels. In a first step, we will derive the first-best solution as a benchmark. In a second step, we will determine the market solution before analyzing the influence of different public policy programs on contract design.

\subsection{The First-best Solution}

A first necessary step in the evaluation of public policy programs is to define the (public) first-best solution as a reference case. Let us define the entire social value of the project $W(\theta)$ according to the state of the project $\theta$ with $\theta \in\{$ bad, medium, good $\}$ as the sum of the net value of the project and the spill-over effect:

$$
W(\theta)=V(e, a, \alpha, \theta)-\frac{1}{2} \beta e^{2}-\frac{1}{2} \gamma a^{2}-I+B+S(e, a, \alpha)
$$

The public first-best efficient effort levels for the three different states are given by:

$$
\begin{aligned}
e_{b}^{* S P}=0 \quad \text { and } a_{b}^{* S P} & =\frac{1}{\gamma} \frac{\partial l(a)}{\partial a} \\
e_{m}^{* S P}=0 & \text { and } a_{m}^{* S P}=0 \\
e_{g}^{* S P}=\frac{(1+\varepsilon)}{\beta} \frac{\partial \bar{x}(e, a, \alpha)}{\partial e} & \text { and } a_{g}^{* S P}=\frac{(1+\varepsilon)}{\gamma} \frac{\partial \bar{x}(e, a, \alpha)}{\partial a}
\end{aligned}
$$

In addition, it is efficient to give the $\mathrm{E}$ control rights in at least one period in order to conserve the private benefits $B$. As regards the investment decision, an investment is only profitable from a social point of view if it is sufficiently innovative, i.e. $\alpha \geq \underline{\alpha}^{S P}$ 
determined by the following equation:

$$
\begin{aligned}
\bar{x}\left(e_{g}^{* S P}, a_{g}^{* S P}, \underline{\alpha}^{S P}\right)-\frac{1}{2} \beta e_{g}^{* S P^{2}}-\frac{1}{2} \gamma a_{g}^{* S P^{2}}+\frac{B}{p q}+S\left(e_{g}^{* S P}, a_{g}^{* S P}, \underline{\alpha}^{S P}\right)= \\
I+\frac{(1-p)(1-q)}{p q}\left[I-L\left(a_{b}^{* S P}\right)\right]
\end{aligned}
$$

with $L\left(a_{b}^{* S P}\right)=l\left(a_{b}^{* S P}\right)-\frac{1}{2} \gamma a_{b}^{* S P^{2}}$ and $S\left(e_{g}^{* S P}, a_{g}^{* S P}, \underline{\alpha}^{S P}\right)$ being the spill-over effect of a project with the inherent innovative value $\underline{\alpha}^{S P}$ if public first-best effort levels are chosen.

In a second step, we must determine the private first-best solution. To this end, we understand the first-best solution as having a spill-over effect of zero, as the VC and the E will never consider the spill-over effect, though they may be able to solve the adverse selection and moral hazard problems. In this case, the private first-best efficient effort levels for the three different states are given by:

$$
\begin{aligned}
e_{b}^{*}=0 \quad \text { and } \quad a_{b}^{*} & =\frac{1}{\gamma} \frac{\partial l(a)}{\partial a} \\
e_{m}^{*}=0 & \text { and } a_{m}^{*}=0 \\
e_{g}^{*}=\frac{1}{\beta} \frac{\partial \bar{x}(e, a, \alpha)}{\partial e} & \text { and } a_{g}^{*}=\frac{1}{\gamma} \frac{\partial \bar{x}(e, a, \alpha)}{\partial a}
\end{aligned}
$$

A comparison of the private and the public first-best effort levels shows an underinvestment in effort of both contracting parties in the good state of the project. In the medium and bad states of the project, effort levels are identical: $a_{b}^{*}=a_{b}^{* S P}$ and $a_{m}^{*}=a_{m}^{* S P}$ as well as $e_{b}^{*}=e_{b}^{* S P}$ and $e_{m}^{*}=e_{m}^{* S P}$. In order to simplify notation, we will use the first representation in these two states.

Moreover, it is still efficient to give control rights to the $\mathrm{E}$ in at least one period. As regards the investment decision, we know that the project is only profitable from a private point of view if $\alpha \geq \underline{\alpha}^{P R}$ determined by the following equation:

$$
\bar{x}\left(e_{g}^{*}, a_{g}^{*}, \underline{\alpha}^{P R}\right)-\frac{1}{2} \beta e_{g}^{* 2}-\frac{1}{2} \gamma a_{g}^{* 2}+\frac{B}{p q}=I+\frac{(1-p)(1-q)}{p q}\left[I-L\left(a_{b}^{*}\right)\right]
$$


By comparing equations (8) and (10), we see that $\underline{\alpha}^{P R}$ must be higher than $\underline{\alpha}^{S P}$ as $\bar{x}(e, a, \alpha)$ is increasing in $\alpha$. This means that less innovative projects which are still socially profitable are not profitable for the economic agents and will not be financed ${ }^{4}$.

\subsection{The Market Solution}

It is important to know whether the described private first-best solution can be achieved by the market and, if so, how the contract is to be designed. Hence, as a first step, we will see if a contract exists which induces private first-best effort levels of both contracting parties and if the E has an incentive to choose this contract; and in a second step, we will check if all privately profitable projects are financed.

Incentives in the good state can only be given by a compensation scheme which depends on the realized outcome. Thus debt cannot be efficient in the good state of the project - however, it is in the bad state because only the VC has to get an incentive to exert effort. Equity, on the other hand, gives suboptimal incentives in both bad and good states because it is impossible to make both parties full residual claimants at the same time. These problems cannot be solved by debt-equity mixes either. However, following the insights of Schmidt [2003], we show that adequately designed preferred stock contracts also provide efficient incentives to both contracting parties in our sequential double-sided moral hazard framework.

Proposition 1 The E offers the VC a contract of convertible preferred stock which implements the private first-best situation. This contract guarantees the VC a limited preferred dividend of $D$ and the right to convert the preferred stock into an equity stake $s_{1}^{C V}$ in $t_{4}$

\footnotetext{
${ }^{4}$ We further assume that $\bar{x}\left(e_{g}^{*}, a_{g}^{*}, \alpha\right)-\frac{1}{2} \beta e_{g}^{* 2}-\frac{1}{2} \gamma a_{g}^{* 2}+\frac{B}{p q} \leq x\left(e_{g}^{*}, \delta, \alpha\right)$ and $(1+\varepsilon) \bar{x}\left(e_{g}^{* S P}, a_{g}^{* S P}, \alpha\right)-$ $\frac{1}{2} \beta e_{g}^{* S P^{2}}-\frac{1}{2} \gamma a_{g}^{* S P^{2}}+\frac{B}{p q} \leq(1+\varepsilon) x\left(e_{g}^{* S P}, \delta, \alpha\right)$. This assumption ensures that the basic mechanism of the convertible security works for all privately (socially) profitable projects. Otherwise, the range of projects which get financed would be smaller as private (social) first-best effort levels would not be implementable for all projects. Details see appendix A1.
} 
if the project's surplus reaches at least the threshold $z$ :

$$
\begin{aligned}
D & =C=I+\frac{(1-p)(1-q)}{p q}\left[I-L\left(a_{b}^{*}\right)\right] \\
s_{1}^{C V} & =\frac{C+0,5 \gamma a_{g}^{* 2}}{\left[\bar{x}\left(e_{g}^{*}, a_{g}^{*}, \alpha\right)\right]} \\
z & =\bar{x}\left(e_{g}^{*}, a_{g}^{*}, \alpha\right)
\end{aligned}
$$

Proof 1 See appendix.

The idea behind proposition 1 is as follows. Convertible preferred stock contracts are able to implement the private first-best situation because they combine the advantages of both debt and equity: they function as pure debt contracts in the bad and medium states of the project, that which is efficient, yet, the conversion option simultaneously guarantees an efficient outcome in the good state. This is due to the following: the VC is a full residual claimant in the bad state and thus chooses the efficient effort level, while in the good state when debt contracts are no longer efficient, the threshold $z$ guarantees that the VC will not choose a suboptimal effort level, and the sequential effort decision structure guarantees that the $\mathrm{E}$ has an incentive to choose the threshold $z$ in such a way that he also has an incentive to choose his private first-best effort level. As private firstbest effort levels can be induced in this way without paying a rent to the VC, the E's profit is maximized. Finally, as the limited liability constraint is not binding, all privately profitable projects get financed.

\subsection{The Influence of Public Intervention on Contract Design}

In the previous subsection, we pointed out that the market is able to solve the problems of moral hazard and adverse selection by using adequately designed financial instruments, namely convertible preferred stock contracts. Thus the private first-best situation 
is reached allowing public policy programs to focus on internalizing the spill-over effect due to successful innovation. As a broad range of public policy programs exists, it is important to know their effects on contract design and the individual behavior if suitable public policy measures are to be identified.

Below, we will look at five different public policy categories: ex post grants, guarantee programs, ex ante grants, investment grants and public support. In doing so, we will determine the design of each public policy measure necessary in order to get the highest possible percentage of socially profitable projects financed ${ }^{5}$. Given this public policy measure, we will derive the optimal contract design between the E and the VC. Here, it is important to bear in mind that we assume that the contracting parties are not able to write any side contracts because the public institution requires all contractual documents related to the relationship in order to avoid misuse of support programs - a fact that is frequently observed in practice. Moreover, in order to concentrate on our analysis, we assume lump-sum financing of public policy programs. Finally, we need to make an assumption about the observability and verifiability of the inherent innovative value $\alpha$ of each project. For the following analysis, we assume that it is not observable ex ante by the public institution. In section 5, we will expand on two alternative assumptions. However, it is important to consider that an ex post discrimination is always possible due to the verifiability of the generated surplus.

\section{Ex post Grants}

Ex post grants can be made contingent on the outcome of the project. Therefore, the grant can be paid to the E only when a spill-over effect exists and furthermore, the grant

\footnotetext{
${ }^{5}$ For the sake of simplification, we assume that the spill-over effect exceeds a specific minimum level: $S\left(e_{g}^{*}, a_{g}^{*}, \underline{\alpha}^{P R}\right)>\frac{(1-p)(1-q)}{p q}\left[L\left(a_{b}^{*}\right)-l(0)\right]$. This assumption guarantees that all projects that are privately profitable without intervention are at least socially profitable with intervention, even when taking into account possible lower effort levels resulting from the public intervention itself.
} 
can be proportional to the actual outcome. Now the question is what influence this policy instrument has on the contract mechanisms and whether it is possible to achieve the public first-best situation.

We know that whereas in the bad and medium states, nothing changes as the E does not receive a grant, in the good state, the cash-flow of the project increases. Furthermore, we have shown in proof 1 that with adequately designed convertible securities, the E will get the project's net surplus and has therefore an incentive to implement private first-best effort levels. As the amount of the ex post grant equally depends on the effort levels of both contracting parties, the E will get an incentive to further increase the effort levels.

Indeed, if we assume that the public institution pays an ex post grant of $\zeta \bar{x}(e, a, \alpha)$ for $V>I$ and solve the E's modified maximization problem analogously to proof 1 , we get the following results: all incentive constraints are still fulfilled for $D=C$ - i.e. the basic mechanism of the convertible security remains unchanged - and the effort levels which maximize the E's profit now amount to

$$
\begin{aligned}
\underline{e}^{*} & =\frac{(1+\zeta)}{\beta} \frac{\partial \bar{x}(\underline{e}, \underline{a}, \alpha)}{\partial \underline{e}} \\
\underline{a}^{*} & =\frac{(1+\zeta)}{\gamma} \frac{(1+\zeta) \partial \bar{x}(\underline{e}, \underline{a}, \alpha)}{\partial \underline{a}}
\end{aligned}
$$

So in order to induce public first-best effort levels $\zeta$ must be equal to $\varepsilon$. Then the $\mathrm{E}$ modifies the threshold to $z_{E P G}=\bar{x}\left(e_{g}^{* S P}, a_{g}^{* S P}, \alpha\right)$ in order to implement public first-best effort levels. Moreover, he adapts the conversion stake in order to guarantee that the VC's participation constraint continues to be binding, i.e.

$$
s_{1, E P G}^{C V}=\frac{C+\frac{1}{2} \gamma a_{g}^{* S P^{2}}}{(1+\varepsilon) \bar{x}\left(e_{g}^{* S P}, a_{g}^{* S P}, \alpha\right)} \text { with } C=I+\frac{(1-p)(1-q)}{p q}\left[I-L\left(a_{b}^{*}\right)\right]
$$

This implies that the E's profit corresponds to the net social surplus of the project and thus the E's participation constraint is fulfilled for all socially profitable projects. As the E's limited liability constraint is not binding, the public first-best situation is achieved. 
We can sum up our results in the following proposition:

Proposition 2 Paying the $E$ an ex post grant $E P G=\varepsilon \bar{x}(e, a, \alpha)$ for $V>I$ gives the $E$ an incentive to offer modified preferred stock contracts which induce public first-best effort levels. All socially profitable projects get financed. Welfare is maximized.

Proof 2 Analogous to proof 1 taking into account the modifications mentioned.

\section{Guarantee Programs}

As already pointed out, there are (were) many public policy programs of which the purpose is (was) to promote $\mathrm{VC}$ financing by reducing the possible loss for the $\mathrm{VC}$ in case of failure. For simplification, we assume that risk is totally taken on by the public institution, i.e. the VC gets back her initial investment $I$ even if the project is in the bad state. We again study the effect of this policy instrument on contract design and the resulting market outcome.

We know that whereas in the good and medium states nothing changes, as the initial investment amount is always guaranteed, in the bad state the payoff of the $\mathrm{VC}$ now amounts to

$$
\Pi_{b, G P}^{V C}=I-\frac{1}{2} \gamma a^{2}
$$

while the monetary payoff of the E continues to be 0 . This change has a crucial impact on the VC's incentives as she will now choose an effort level of 0 in order to maximize her payoff. At the same time, this implies that the expected social net value of all projects that would have been financed without intervention is reduced to $E[W(\alpha)]$ with:

$$
\begin{aligned}
E[W(\alpha)]=p q\left[\bar{x}\left(e_{g}^{*}, a_{g}^{*}, \alpha\right)-\right. & \left.\frac{1}{2} \beta e_{g}^{* 2}-\frac{1}{2} \gamma a_{g}^{* 2}+S\left(e_{g}^{*}, a_{g}^{*}, \alpha\right)\right] \\
& +[(1-p) q+(1-q) p] I+(1-p)(1-q) l(0)+B-I
\end{aligned}
$$


Furthermore, the least innovative socially profitable project has also to be more innovative than in a public first-best situation. In fact, the least innovative project which is still socially profitable under these conditions has only an innovative value of $\underline{\alpha}_{a_{b}=0}$ given by $E\left[W\left(\underline{\alpha}_{a_{b}=0}\right)\right]=0$ for which $\underline{\alpha}^{P R}>\underline{\alpha}_{a_{b}=0}>\underline{\alpha}^{S P}$ holds.

As the guarantee program ensures the VC her initial investment in the bad state of the project but does not change the payoff structures otherwise, the E's maximization problem is only slightly modified. Analogous to proof 1, it can be shown that the incentive constraints continue to hold for $D=C$. Thus the basic mechanism of the convertible security remains unchanged: the E still chooses $z_{G P}=\bar{x}\left(e_{g}^{*}, a_{g}^{*}, \alpha\right)$ in order to implement private first-best effort levels. The only difference is that the $\mathrm{E}$ has an incentive to adapt the conversion stake in such a way that the VC's participation constraint continues to be binding, i.e.

$$
s_{1, G P}^{C V}=\frac{C+\frac{1}{2} \gamma a_{g}^{* 2}}{\bar{x}\left(e_{g}^{*}, a_{g}^{*}, \alpha\right)} \text { with } C=I
$$

This implies that the E's expected profit increases to:

$$
\Pi_{G P}^{E}(\alpha)=p q\left[\bar{x}\left(e_{g}^{*}, a_{g}^{*}, \alpha\right)-\frac{1}{2} \beta e_{g}^{* 2}-\frac{1}{2} \gamma a_{g}^{* 2}-I\right]+B
$$

Since - just as in proof 1 - the E's limited liability constraint is not binding, all projects for which $\Pi_{G P}^{E} \geq 0$ will get financed. When we compare the socially optimal investment decision (see 14) with the private one, we see that if

$$
S\left(e_{g}^{*}, a_{g}^{*}, \underline{\alpha}_{G P}\right)=\frac{(1-p)(1-q)}{p q}[I-l(0)]
$$

holds, all socially profitable projects under these conditions get financed. If condition (17) holds only with $>$, more but not all socially profitable projects will get financed and if it holds only with $<$, even socially unprofitable projects will get financed.

The following proposition summarizes our results: 
Proposition 3 Guarantee programs bring about a decrease in the effort level of the VC to 0 in the bad state of the project, implying a worsened outcome for all projects that would have been financed without introduction of the guarantee program. More, but not all, socially profitable projects (under these conditions) are financed if the spill-over effect is relatively large. In the reverse case, even socially unprofitable projects get financed. The total welfare effect depends on the parameters.

Proof 3 Analogous to proof 1 taking into account the modifications mentioned.

\section{Ex ante Grants}

It is important to distinguish between ex post and ex ante grants as the former can depend on the outcome while the latter are just fixed support payments. Thus ex ante grants do not give any further incentives in medium and good states but they do relax the E's participation and limited liability constraints in all states. Therefore, the impact of this measure is somewhat similar to that of guarantee programs.

In fact, as ex ante grants are fixed support payments, they may only change the VC's incentives in the bad state. Thereby, their impact depends on the size of the ex ante grant. If the ex ante grant is relatively large (i.e. $E A G>D-l(0)$ ), the VC's claim is guaranteed independently of her effort level and thus she chooses $a=0$. For a medium-sized ex ante grant (i.e. $\left.D-l\left(a_{b}^{*}\right) \leq E A G<D-l(0)\right)$, the VC chooses $a=\hat{a}_{b}^{E A G} \in\left(0, a_{b}^{*}\right]$ so that her

claim is just guaranteed, i.e. $l\left(\hat{a}_{b}^{E A G}\right)+E A G=D$. Finally, with a relatively small ex ante grant $\left(E A G<D-l\left(a_{b}^{*}\right)\right)$, the $\mathrm{VC}$ always gets the total surplus of the project in the bad state and so has an incentive to invest efficiently.

As ex ante grants do not change incentives in medium and good states, the basic mechanism of the convertible security remains unchanged. Indeed, analogous to proof 1 , 
it can be shown that the $\mathrm{E}$ still has an incentive to choose $z_{E A G}=\bar{x}\left(e_{g}^{*}, a_{g}^{*}, \alpha\right)$ in order to induce private first-best effort levels of both contracting parties in the good state. Moreover, the incentive constraints continue to be fulfilled for $D=C$. However, as the outcome of the project is higher in all states and thus the E's limited liability constraints are relaxed, the VC's claim in the good state has to be adapted to

$$
s_{1, E A G}^{C V}=\frac{C+\frac{1}{2} \gamma a_{g}^{* 2}}{\bar{x}\left(e_{g}^{*}, a_{g}^{*}, \alpha\right)+E A G}
$$

in order to ensure that her participation constraint continues to be binding. The VC's claim $C$ depends on the size of the ex ante grant. We have to distinguish between the three cases mentioned:

Case 1: $E A G>D-l(0)$ : The VC's initial investment is always guaranteed. Thus a binding $\mathrm{PC}(\mathrm{VC})$ requires $C_{1}^{E A G}=D=I$.

Case 2: $D-l\left(a_{b}^{*}\right)<E A G<D-l(0)$ : The VC's initial investment is only guaranteed if the VC chooses an effort level of $\hat{a}_{b}^{E A G} \in\left(0, a_{b}^{*}\right]$. As $l\left(a_{b}^{*}\right)<I, D$ is always guaranteed in the medium state. A binding $\mathrm{PC}(\mathrm{VC})$ requires $C_{2}^{E A G}=I+(1-$ $p)(1-q) \frac{1}{2} \gamma a_{b}^{\text {dev }}{ }^{2}$

Case 3: $E A G \leq D-l\left(a_{b}^{*}\right)$ : The claim of the $\mathrm{VC}$ is never guaranteed in the bad state. As regards the medium state, we have to distinguish two subcases:

Subcase a: $E A G>D-I$ : Here the claim is always guaranteed in the medium state and a binding $\mathrm{PC}(\mathrm{VC})$ demands $C_{3 a}^{E A G}=I+\frac{(1-p)(1-q)}{1-(1-p)(1-q)}\left[I-L\left(a_{b}^{*}\right)-E A G\right]$

Subcase b: $E A G<D-I$ : Here the claim is never guaranteed in the medium state and a binding $\mathrm{PC}(\mathrm{VC})$ demands $C_{3 b}^{E A G}=I+\frac{(1-p)(1-q)}{p q}\left[I-L\left(a_{b}^{*}\right)\right]-\frac{1-p q}{p q} E A G$. 
We can see that the larger the ex ante grant, the smaller the VC's claim C.

As far as the socially optimal investment decision with the introduction of the ex ante grant is concerned, we know that the least innovative project which is still socially profitable depends on the effort level of the VC in the bad state $\left(\hat{a}_{E A G}^{i}\right)$ and is determined by the following equation:

$$
\begin{aligned}
\bar{x}\left(e_{g}^{*}, a_{g}^{*}, \underline{\alpha}_{a_{b}=\hat{a}_{E A G}^{i}}\right)-\frac{1}{2} \beta e_{g}^{* 2}-\frac{1}{2} \gamma a_{g}^{* 2}+\frac{B}{p q}= \\
I+\frac{(1-p)(1-q)}{p q}\left[I-L\left(a^{i}\right)\right]-S\left(e_{g}^{*}, a_{g}^{*}, \underline{\alpha}_{a_{b}=\hat{a}_{E A G}^{i}}\right)
\end{aligned}
$$

In comparison, the private investment decision is determined by the E's participation as well as his limited liability constraint in the good state. In the basic case without intervention his limited liability constraint is automatically fulfilled when his participation constraint holds. However, as with ex ante grants, the E may even receive profits in bad and medium states (see cases 1 and 2 as well as subcase 3a), his limited liability constraint may become binding in good states instead. This is the more probable, the higher the E's expected profits in bad and medium states $(E(Y))$ and the lower the difference between the E's effort costs in the good state and his private benefit $A=p q \frac{1}{2} \beta e_{g}^{* 2}-B$. If the E's limited liability constraint is binding in the good state, a project must exceed the critical innovative value $\underline{\alpha}_{a_{b}=\hat{a}_{E A G}^{i}}$ given by:

$$
\bar{x}\left(e_{g}^{*}, a_{g}^{*}, \underline{\alpha}_{a_{b}=\hat{a}_{E A G}^{i}}\right)-\frac{1}{2} \gamma a_{g}^{* 2}=C_{i}^{E A G}-E A G
$$

in order to get financed. Consequently, in these cases, the public institution has to pay the following ex ante grant in order to make private and social minimum levels of the inherent innovative value coincide:

$$
E A G=S\left(e_{g}^{*}, a_{g}^{*}, \underline{\alpha}\right)-\left[I+\frac{(1-p)(1-q)}{p q}\left[I-L\left(a^{i}\right)\right]-C_{i}^{E A G}\right]-\frac{A}{p q}
$$


If the participation constraint is binding, the range of privately profitable projects is determined by:

$$
\bar{x}\left(e_{g}^{*}, a_{g}^{*}, \underline{\alpha}_{a_{b}=\hat{a}_{E A G}^{i}}\right)-\frac{1}{2} \beta e_{g}^{* 2}-\frac{1}{2} \gamma a_{g}^{* 2}+\frac{B}{p q} \geq C_{i}^{E A G}-E A G-\frac{1}{p q} E(Y)
$$

In this case, the optimal amount of the ex ante grants must correspond to

$$
E A G=S\left(e_{g}^{*}, a_{g}^{*}, \underline{\alpha}_{a_{b}=a_{b}^{*}}\right)-\left[I+\frac{(1-p)(1-q)}{p q}\left[I-L\left(a^{i}\right)\right]-C_{i}^{E A G}\right]-\frac{1}{p q} E(Y)
$$

in order to get all socially profitable projects financed. The optimal ex ante grants as well as the resulting optimal contracts for the different cases as well as for different levels of $A$ are given in the appendix (see table 8.3).

The welfare impact of ex ante grants depends on their size which determines the outcome in the bad state depending on the size of the spill-over effect. According to table 8.3

- for a small A, the effort level of the VC is lower than the efficient level if $S\left(e_{g}^{*}, a_{g}^{*}, \underline{\alpha}^{P R}\right)>$

$$
\left[I-l\left(a_{b}^{*}\right)\right]+\frac{(1-p)(1-q)}{p q}\left[I-L\left(a_{b}^{*}\right)\right]+\frac{A}{p q}
$$

- for a larger $\mathrm{A}$, this is the case for

$$
S\left(e_{g}^{*}, a_{g}^{*}, \underline{\alpha}^{P R}\right)>\frac{1}{p q}\left[I-l\left(a_{b}^{*}\right)\right]+\frac{(1-p)(1-q)}{p q}\left[\frac{1}{2} \gamma a_{b}^{* 2}\right] .
$$

To be more precise, the higher the spill-over effect the lower the effort level. If the VC's effort level in the bad state is lower than the efficient one, then the net value of all projects which would have been financed without intervention is reduced. As more projects get financed, the aggregate welfare effect depends on the parameters. If the spill-over effect is smaller, the VC continues to choose his efficient effort level in the bad state and so the aggregate welfare effect is positive. We can sum up our results in the following proposition: 
Proposition 4 Ex ante grants may bring about a decrease in the effort level of the $V C$ in the bad state, implying a worsened outcome for all projects that would have been financed without introduction of the ex ante grant. This effect is the more pronounced, the larger the spill-over effect and does not exist at all for relatively small spill-over effects. All socially profitable projects (under these conditions) get financed. The total welfare effect depends on the parameters for relatively large spill-over effects and is positive for relatively small spill-over effects.

Proof 4 Analogous to proof 1 taking into account the modifications mentioned.

\section{Investment Grants}

Governments also frequently aim to promote innovative companies by channelling further funds into the venture capital industry. Here, we will focus on two forms which are frequently encountered in practice: re-financing and co-financing. Thereby, we will restrict our analysis to the most basic case in order to reveal the underlying mechanisms: the public institution assumes part of the investment costs $I^{*}(\tau I)$ in exchange for a fixed claim $D^{*}=I^{*}$ (equity claim $\tau$ ), i.e. the investment grant does not involve any further costs for the VC. Moreover, we assume that the public institution does not provide any know-how neither to the $\mathrm{VC}$ nor directly to the $\mathrm{E}^{6}$.

Finally, we will describe two cases as regards the distribution of the liquidation value in the bad state. On the one hand, we assume that the liquidation value is divided between the contracting parties proportionally to their contribution to the investment costs; on the other hand, we assume that the $\mathrm{VC}$ has a form of liquidation preference which guarantees that she preferably gets back her contribution to the investment costs. As the project's

\footnotetext{
${ }^{6}$ We will analyze this form of additional support separately in the next subsection.
} 
outcome $V(e, a, \alpha, \theta) \geq I$ for $\theta=\{$ medium, good $\}$, we assume that the public institution's claim has always to be fulfilled by the VC in these states.

We will begin by determining the influence of re-financing schemes. Analogous to proof 1 , we can first determine the choice of effort levels. Here, it is important to note that the public institution does not negotiate directly with the $\mathrm{E}$ but that the $\mathrm{VC}$ continues to negotiate the whole contract with the E while subcontracting with the public institution. As the VC continues to be the full residual claimant of the E's payment in the good state (the public institution only receives a fixed payment which is always smaller in equilibrium than the total payment of the E), the basic mechanism of the convertible security remains unchanged. This means that the $\mathrm{E}$ will still choose $z_{E A G}=\bar{x}\left(e_{g}^{*}, a_{g}^{*}, \alpha\right)$ in order to induce private first-best effort levels of both contracting parties and that all incentive constraints continue to be fulfilled for $D=C$.

In the bad state of the project, however, the VC's effort choice depends on the design of the support program. If the $\mathrm{VC}$ holds a liquidation preference, we have to distinguish between three cases:

1. $I-I^{*} \geq l\left(a_{b}^{*}\right)$ : In this case, the VC gets the total surplus of the project in the bad state what implies that she continues to choose $a_{b}^{*}$.

2. $l(0)<I-I^{*}<l\left(a_{b}^{*}\right)$ : In this case, the VC must choose only $\hat{a}_{b}^{I G 1-L P} \in\left(0, a_{b}^{*}\right)$ in order to guarantee her claim. Thus she will underinvest in effort.

3. $I-I^{*} \leq l(0)$ : In this case, the VC's claim is guaranteed independently of her effort level and thus she will choose $a_{b}=0$.

Furthermore, we have to take into account the VC's modified participation constraint which the E still wants to be binding. Thereby, the modified claim $C$ of the VC depends 
on the size of the investment costs assumed by the public institution and amounts for the three cases mentioned to:

$$
\begin{aligned}
& C_{1}^{I G 1-L P}=I+\frac{(1-p)(1-q)}{p q}\left[I-I^{*}-L\left(a_{b}^{*}\right)\right] \\
& C_{2}^{I G 1-L P}=I+\frac{(1-p)(1-q)}{p q}\left[I-I^{*}-L\left(\hat{a}_{b}^{I G 1-L P}\right)\right] \\
& C_{3}^{I G 1-L P}=I
\end{aligned}
$$

As the E's limited liability constraint in the good state is automatically fulfilled when his participation constraint holds, all projects for which the following condition is valid will get financed:

$$
\bar{x}\left(e_{g}^{*}, a_{g}^{*}, \alpha\right)-\frac{1}{2} \beta e_{g}^{* 2}-\frac{1}{2} \gamma a_{g}^{* 2}+\frac{B}{p q} \geq C_{I G 1-L P}^{i}
$$

This implies that in order to get all socially profitable projects financed, the public institution must assume

$$
I_{L P}^{*}=\frac{p q}{(1-p)(1-q)} S\left(e_{g}^{*}, a_{G}^{*}, \underline{\alpha}_{a_{b}=\hat{a}_{I G 1-L P}^{i}}\right)
$$

the innovative value of the least innovative but still socially profitable project $\underline{\alpha}_{a_{b}=\hat{a}_{I G 1-L P}^{i}}$ depending on the effort level chosen by the VC in the bad state. Note, however, that for $S\left(e_{g}^{*}, a_{g}^{*}, \underline{\alpha}_{a_{b}=0}\right)>\frac{(1-p)(1-q)}{p q}[I-l(0)]$, the claim $C$ no longer depends on the size of $I^{*}$. Therefore, in this case, it will never be possible to induce financing of all socially profitable projects and the public institution will choose $I^{*}=[I-l(0)]$ irrespective of the extent of the spill-over effect.

If the $\mathrm{VC}$ does not hold a liquidation preference but the claims of both contracting parties in the bad state are equally ranked, the $\mathrm{VC}$ will always underinvest in effort in the bad state. In fact, she will choose the effort level which maximizes her payoff: $\hat{a}_{b}^{I G 1-N L P}=\frac{I-I^{*}}{I \gamma} \frac{\partial l(a)}{\partial a}$. Thus we have to determine the modified claim $C$ which guarantees 
that the VC's participation constraint continues to be binding:

$$
C^{I G 1-N L P}=I+\frac{(1-p)(1-q)}{p q}\left[I-I^{*}-L\left(\hat{a}_{b}^{I G-N L P}\right)+\frac{I^{*}}{I} l\left(\hat{a}_{b}^{I G 1-N L P}\right)\right]
$$

Analogous to the case with a liquidation preference, the optimal contribution of the public institution now amounts to

$$
I_{N L P}^{*}=\frac{p q}{(1-p)(1-q)\left[1-\frac{l\left(\hat{a}_{b}^{I G 1-N L P}\right)}{I}\right]} S\left(e_{g}^{*}, a_{g}^{*}, \underline{\alpha}_{a_{b}=\hat{a}_{I G 1-N L P}}\right)
$$

the innovative value of the least innovative but still socially profitable projects $\underline{\alpha}_{\hat{a}_{b}^{I G 1-N L P}}$ increasing in $I^{*}$. However, as $I^{*} \leq I$, for $S\left(e_{g}^{*}, a_{g}^{*}, \underline{\alpha}_{a_{b}=0}\right)>\frac{(1-p)(1-q)}{p q}[I-l(0)]$, all socially profitable projects never get financed.

Now, we will have a closer look at co-financing schemes. As the public institution receives $\tau$ of the effort compensation in the good state, we will further assume that it compensates the $\mathrm{VC}$ for this forgone effort costs, i.e. an additional agreement exists which reduces the claim of the public institution by $\tau \frac{1}{2} \gamma \underline{a}^{2}$ whenever conversion takes place. This implies that analogous to the case of re-financing schemes the underlying mechanism of the convertible security remains unchanged ${ }^{7}$.

Thus we can again limit our analysis to the effects of the two forms of co-financing schemes mentioned in the bad state of the project. If the VC holds a liquidation preference, we have to distinguish between three analogous cases:

1. $(1-\tau) I \geq l\left(a_{b}^{*}\right):$ In this case, the VC never receives her full claim what implies that she gets the whole liquidation value. In turn, she continues to choose $a_{b}^{*}$.

2. $l(0)<(1-\tau) I<l\left(a_{b}^{*}\right)$ : In this case, the VC must choose only $\hat{a}_{b}^{I G 2-L P} \in\left(0, a_{b}^{*}\right)$ in order to guarantee her claim. Thus she will underinvest in effort.

\footnotetext{
${ }^{7}$ Otherwise, the $\mathrm{E}$ would have to adjust the conversion stake in order to be able to implement the convertible security and the outcome would always be worse than with our simplifying assumption.
} 
3. $(1-\tau) I \leq l(0)$ : In this case, the VC's claim is guaranteed independently of her effort level and thus she will choose $a_{b}=0$.

If the $\mathrm{VC}$ does not hold a liquidation preference, she will maximize her outcome in the bad state by choosing $\hat{a}_{b}^{I G 2-N L P}=\frac{(1-\tau)}{\gamma} \frac{\partial l(a)}{\partial a}$. For all cases we then need to determine the claim $C$ which is necessary in order to guarantee that the VC's participation constraint is binding:

$$
\begin{aligned}
C_{1}^{I G 2-L P} & =I+\frac{(1-p)(1-q)}{p q}\left[I-\frac{L\left(a_{b}^{*}\right)}{1-\tau}\right] \\
C_{2}^{I G 2-L P} & =I+\frac{(1-p)(1-q)}{p q}\left[I-\frac{L\left(\hat{a}_{b}^{I G 1-L P}\right)}{1-\tau}\right] \\
C_{3}^{I G 2-L P} & =I \\
C^{I G 2-N L P} & =I+\frac{(1-p)(1-q)}{p q}\left[I-L\left(\hat{a}_{b}^{I G-N L P}\right)+\frac{\tau}{(1-\tau)} \frac{1}{2} \gamma\left(\hat{a}_{b}^{I G 1-N L P}\right)^{2}\right]
\end{aligned}
$$

Analogous to the case of re-financing schemes, comparing the private with the socially optimal financing decision yields the optimal $\tau^{*}$. As concerns the case without a liquidation preference, we see that $C^{I G 2-N L P}$ is always larger than the claim $C=I+\frac{(1-p)(1-q)}{p q}[I-$ $\left.L\left(a_{b}^{*}\right)\right]$ without intervention. This implies that less projects get financed and that therefore, co-financing schemes without a liquidation preference should never be implemented in this basic form. As regards the implementation with a liquidation preference, the optimal $\tau$ is given by

$$
\tau=\frac{S\left(e_{g}^{*}, a_{g}^{*}, \underline{\alpha}_{a_{b}=\hat{a}_{I G 2-L P}^{i}}\right)}{S\left(e_{g}^{*}, a_{g}^{*}, \underline{\alpha}_{a_{b}=\hat{a}_{I G 2-L P}^{i}}\right)+\frac{(1-p)(1-q)}{p q} L\left(a_{b}^{I G 2-L P_{i}}\right)}
$$

for case 1 and 2 . In case 3 , the value of $\tau$ does not have any influence on the financing decision. This implies that for $S\left(e_{g}^{*}, a_{g}^{*}, \underline{\alpha}_{a_{b}=0}\right)>\frac{(1-p)(1-q)}{p q}[I-l(0)]$, all socially profitable projects never get financed and $\tau^{*}=\frac{I-l(0)}{I}$ irrespective of the extent of the spill-over effect.

Thus, we are able to show that the impact of investment grants greatly depends on 
the concrete design of the program. The proposition summarizes our results:

Proposition 5 If the VC holds a liquidation preference in the bad state, both types of investment grants are welfare-increasing if the spill-over effect is sufficiently small. If the spill-over effect is medium (large), all (more) socially profitable projects (under the corresponding conditions) are financed but now the outcome of all projects which would have been financed without the introduction of the investment grant is reduced due to the negative incentive effect in the bad state. Thus the total welfare effect depends on the parameters.

If the VC does not hold a liquidation preference, investment grants always have a negative incentive effect in the bad state. As with re-financing schemes all, or at least more socially profitable projects are financed, the total welfare effect depends again on the parameters. With co-financing schemes, however, the project range is not enlarged. Thus they are welfare-decreasing in this case.

Proof 5 Analogous to proof 1 taking into account the modifications mentioned.

\section{Public Support}

Public support, such as technical assistance or access to networks, may decrease the effort costs of VCs and Es. As its objective is to internalize the spill-over effect, the public institution aims at decreasing the VC's costs for managerial contributions as well as the E's costs for technological development but not the VC's costs for selling off the assets. Moreover, public support should only bring about an effect while the measure is in force and thus training programs which aim at ultimately reducing the costs are not suitable for internalizing the spill-over effect.

This means that the public institution supports both contracting parties by reducing 
their effort costs in the good state to

$$
\begin{aligned}
& c(e)_{P S}=\frac{1}{2} \kappa \beta e^{2} \\
& c(a)_{P S}=\frac{1}{2} \kappa \gamma a^{2}
\end{aligned}
$$

Taking into account these modified effort costs and solving the E's modified maximization problem analogous to proof 1 yields the following results: the incentive constraints are still fulfilled for $D=C^{8}$ - i.e. the basic mechanism of the convertible security remains unchanged - and the effort levels which maximize the E's profit now amount to

$$
\begin{aligned}
\underline{e}^{*} & =\frac{1}{\kappa \beta} \frac{\partial \bar{x}(\underline{e}, \underline{a}, \alpha)}{\partial \underline{e}} \\
\underline{a}^{*} & =\frac{1}{\kappa \gamma} \frac{\partial \bar{x}(\underline{e}, \underline{a}, \alpha)}{\partial \underline{a}}
\end{aligned}
$$

We can see that the lower $\kappa$, i.e. the higher the support by the public institution, the higher the implemented effort levels will be. Indeed, if $\kappa$ amounts to $\frac{1}{1+\varepsilon}$, the $\mathrm{E}$ has an incentive to choose $z_{P S}=\bar{x}\left(e_{g}^{* S P}, a_{g}^{* S P}, \alpha\right)$ in order to implement public first-best effort levels. Furthermore, the E will adapt the post conversion stake to

$$
s_{1, P S}^{C V}=\frac{C+\frac{1}{2} \frac{\gamma}{1+\varepsilon} a_{g}^{* S P^{2}}}{\bar{x}\left(e_{g}^{* S P}, a_{g}^{* S P}, \alpha\right)} \text { with } C=I+\frac{(1-p)(1-q)}{p q}\left[I-L\left(a_{b}^{*}\right)\right]
$$

in order to ensure that the VC's participation constraint continues to be binding.

As concerns the investment decision, the E's limited liability constraint continues to be automatically fulfilled if the E's participation constraint holds. This is due to the fact that the E's effort costs are higher in equilibrium with public support than without

\footnotetext{
${ }^{8}$ Note, however, that in this case, we need even a more restrictive assumption as concerns the impact of the E's contribution in order to ensure the mechanism of the convertible security (see appendix A1), namely $(1+\varepsilon) \bar{x}\left(e^{* S P}, a^{* S P}, \alpha\right)-\frac{1}{2} \beta e_{g}^{* S P^{2}}-\frac{1}{2} \gamma a_{g}^{* S P^{2}}+(1+\varepsilon) \frac{B}{p q} \leq(1+\varepsilon) x\left(e_{g}^{* S P}, \delta, \alpha\right)$. If this assumption does not hold, the range of financed projects will be smaller as indicated, the actual range depending on the concrete parameter constellation.
} 
intervention. Consequently, the innovative value of the least innovative project which still gets financed $\left(\underline{\alpha}_{P S}\right)$ is given by the following condition:

$$
\begin{aligned}
& \frac{1}{1+\varepsilon}\left[(1+\varepsilon) \bar{x}\left(e_{g}^{* S P}, a_{g}^{* S P}, \underline{\alpha}_{P S}\right)-\frac{1}{2} \beta e_{g}^{* S P^{2}}-\frac{1}{2} \gamma a_{g}^{* S P^{2}}\right]+\frac{B}{p q}= \\
& I+\frac{(1-p)(1-q)}{p q}\left[I-L\left(a_{b}^{*}\right)\right]
\end{aligned}
$$

As regards the socially optimal investment decision with public first-best effort levels in all three stages of the project, we know that all project with $\alpha \geq \underline{\alpha}_{S P}$ get financed (see equation 8). A comparison of both conditions shows that $\underline{\alpha}_{S P}<\underline{\alpha}_{P S}$ : less innovative projects which are still socially profitable do not get financed.

Thus the impact of public support can be summarized as follows.

Proposition 6 Public support implying a cost reduction by the factor $\kappa=\frac{1}{1+\varepsilon}$ for both contracting parties in the good state induces public first-best effort levels of both contracting parties in the good state. More, but not all, socially profitable projects get financed. Public support is thus welfare-increasing.

Proof 6 Analogous to proof 1 taking into account the modifications mentioned.

\section{The Case of Experienced Venture Capital Markets}

Contrary to the previous analysis, in more experienced markets, ex ante information may exist about the expected state of the project. We assume that the information is not freely available but that both agents receive private signals before contracting: the E receives a perfect signal about the product quality and the $\mathrm{VC}$ receives a perfect signal about the market situation. Below, we will show how this altered situation changes our previous results. We proceed analogous to the case of inexperienced markets: in a first step, we 
will derive the first-best solution as a benchmark; in a second step, we will determine the market solution before analyzing the impact of the selected public policy measures on contract design.

\subsection{The First-best Solution}

Analogous to the case of inexperienced markets, we will again determine the public and private first-best solutions as benchmarks. As regards effort levels, nothing changes with respect to the case of experienced markets as both contracting parties also knew the state of the project before choosing their effort levels. This means that, just as in the case of inexperienced markets, private first-best effort levels are suboptimal in the good state of the project. Moreover, it continues to be efficient to give the E control rights in at least one period in order to conserve the private benefits $B$. Moreover, as the true state of the project is known before contracting, the investment decision for each state of the project is taken separately. In the medium and good states, the outcome of the project amounts to at least to the initial investment sum $I$, so that an investment is always profitable; in the bad state, on the other hand, $L\left(a_{b}^{*}\right)+B<I$, and thus, an investment is never socially desirable. It is obvious that the private first-best investment decisions do not differ from the derived public first-best investment decisions because the latter are independent of the size of the spill-over effect.

\subsection{The Market Solution}

In this subsection, we will check - analogous to the case of inexperienced markets whether the derived private first-best solution can be implemented by the market and how the contract will be designed. Therefore, it is important to note that in experienced 
markets both contracting parties before contracting hold private information which they may communicate by acting in a specific way. In the case of the E, this means that he may offer specific menus of contracts depending on the signal he received; and in the case of the $\mathrm{VC}$, this implies that she may communicate her private information by choosing one specific contract or rejecting same. If both parties communicate their private information truthfully, the state of the project can be deduced - if not, the parties must choose their effort levels in a state of uncertainty. Nevertheless, both parties may have incentives to misstate their private information: the E may have an incentive to overstate his private information in order to get bad state projects financed and receive private benefits; the VC may have an incentive to understate her private information in order to get a higher compensation. Therefore, the contract design is crucial; the allocation of control rights, in particular, plays a key role. Indeed, in order to achieve an efficient outcome, a contingent control allocation is necessary. This is due to the fact that private benefits get lost if the $\mathrm{VC}$ holds the control rights and that the $\mathrm{E}$ will always have an incentive to overstate if he holds them independently of the state of the project.

We know from proposition 1 that private first-best effort levels can only be implemented in all states by using adequately designed convertible preferred stock contracts. Following the insights of Houben (2002), we will now show that adequately designed preferred stock contracts also implement this necessary contingent control allocation: they transfer control rights to the $\mathrm{VC}$ but give the $\mathrm{E}$ the possibility to recover them by redeeming the preferred stock in the medium state but not in the bad state of the project. Thus truth-revealing by both agents can be achieved without private benefits being lost.

Proposition 7 The E offers the VC the following truth-revealing contract(s):

- If he receives the bad signal $\underline{\lambda}$, he offers a contract which implies a preferred fixed 
payment $D_{b}$ and which is redeemable at a price $P_{b}$ at $t_{4}$ if control rights are given to the $V C$ :

$$
\begin{aligned}
& D_{b}=I \\
& P_{b}=D_{b}
\end{aligned}
$$

- If he receives the good signal $\bar{\lambda}$, he offers the following menu of contracts:

1. A redeemable voting preferred stock contract: the $V C$ gets control and receives a limited preferred dividend of $D_{m}$, but the $E$ receives the right to redeem the preferred stock at a price $P_{m}$ at $t_{4}$, with

$$
\begin{aligned}
& D_{m}=I \\
& P_{m}=D_{m}
\end{aligned}
$$

2. A convertible preferred stock contract: the $V C$ does not get control, but receives a limited preferred dividend of $D_{g}$ and the right to convert the preferred stock into an equity stake $s_{2}^{C V}$ in $t_{4}$ if the surplus reaches at least the threshold $z$, with

$$
\begin{aligned}
D_{g} & =I \\
s_{2}^{C V} & =\frac{I+0,5 \gamma a_{g}^{* 2}}{\bar{x}\left(e_{g}^{*}, a_{g}^{*}, \alpha\right)} \\
z & =\bar{x}\left(e_{g}^{*}, a_{g}^{*}, \alpha\right) .
\end{aligned}
$$

In addition, these contracts always induce private first-best effort levels.

Proof 7 See appendix.

The idea behind proposition 7 is as follows: As outlined before, the E reveals his private information by offering different contracts to the $\mathrm{VC}$ and truth-revealing is achieved by 
the control transfer of the first contract for high quality projects. Due to this control transfer, the $\mathrm{E}$ does not have an incentive to overstate because he would not receive private benefits in the bad state even if the project got financed. The VC, in turn, reveals her private information by accepting the contract for low quality projects only if she has received the good signal (otherwise, she knows that the project is in the bad state and never profitable), and by choosing the adequate contract according to her received signal within the contract set for high quality projects. Here, truth-revealing by the VC is guaranteed through the threshold $z$. Moreover, we know from proposition 1 that an adequately designed conversion option implements private first-best effort levels in the good state whereas a preferred fixed payment contract is sufficient to achieve private first-best effort levels in bad and medium states.

\subsection{The Influence of Public Intervention on Contract Design}

In the previous subsection, we pointed out that the market is able to solve the doublesided adverse selection as well as the double-sided moral hazard problem. Therefore, public policy can focus on giving further incentives in the good state in order to ensure public first-best effort levels. In what follows, we will analyze the impact of the selected public policy measures. To be more precise, we will take the design of the different public policy measures which was optimal in the case of inexperienced markets and we will determine the impact of these measures on the contract design in experienced markets. This procedure enables us to show that some public policy measures are quite dangerous when the public institution does not know for sure that it is confronted with an inexperienced market. 


\section{Ex post Grants and Public Support}

We know that ex post grants as well as public support do not have any impact on the outcomes of the project in bad and medium states. In good states, on the contrary, the E receives the ex post grant $E P G=\varepsilon \bar{x}(e, a, \alpha)$ or effort costs are reduced by the factor $\kappa=\frac{1}{1+\varepsilon}$. Analogous to the case of inexperienced markets, the $\mathrm{E}$ thus gets the incentive to modify the convertible security in order to implement public first-best effort levels in the good state. As this modification does not change the truth-revealing mechanism, the investment decisions are not influenced by these programs. Consequently, both programs ex post grants and public support - are welfare-maximizing in experienced venture capital markets.

\section{Guarantee Programs, Ex ante Grants and Investment Grants}

We know that guarantee programs, ex ante grants and investment grants do not change the contracting parties' incentives in medium and good states whereas in bad states, incentives may be altered depending partially on the size of the spill-over effect (see section 3.3). However, as shown in subsection 4.2, bad state projects do not get financed in equilibrium in experienced markets without intervention. Consequently, this impact should be irrelevant. Below, we will show, however, that the E may have an incentive to deviate from the derived optimal contract sets in order to get bad state projects financed and receive private benefits of control. In fact, the $\mathrm{E}$ has an incentive to overstate his private information whenever the following two conditions are fulfilled: first, he must gain private benefits in the bad state of the project, and second, the $\mathrm{VC}$ must truthfully reveal her private information when the E offers the same set of contracts irrespective of the signal he receives. This is only possible if a joint contract exists for bad and medium 
states for which the VC's participation constraint is binding.

Without public intervention, this will never be the case in our framework due to the limited liability constraint of the E. It can be achieved, however, through public policy measures which relax the E's limited liability constraint at least in the bad state of the project. With guarantee programs, for example, the VC is protected against losses in bad states. So the E is able to offer a fixed payment contract $D_{m}=I$ which fulfills the VC's participation constraint in bad and medium states. However, it is important that this contract no longer includes a control transfer to the $\mathrm{VC}$ in order to enable the $\mathrm{E}$ to obtain private benefits in the bad state (where he would not be able to redeem the preferred stock contract). This implies that the E will offer the following modified set of contracts, irrespective of the signal he receives:

1. A fixed payment contract which does not give control rights to the VC but only guarantees a fixed payment $D_{m}=I$.

2. A convertible preferred stock contract analogous to proposition 7 .

It can easily be seen that the $\mathrm{VC}$ will continue to truthfully reveal her private information: if she receives the good signal, she may be able to convert, thus she chooses the second contract which also fulfills her participation constraint when conversion is not possible, i.e. in the medium state. If she receives the bad signal, she knows that conversion will never be possible and thus she will choose the first contract. This result is strengthened by the fact that truth-revealing will enable the VC to identify the good state of the project by observing the E's effort level and thus choose her private first-best effort level and induce conversion.

The same is true for investment grants with a liquidation preference of the $\mathrm{VC}$ as well as ex ante grants provided that the spill-over effect is relatively large. This condition is necessary because a fixed payment contract with $D_{m}=I$ only fulfills the VC's partici- 
pation constraint if the VC's claim is guaranteed in the bad state without exerting any effort. Otherwise, the E must pay a mark-up to the VC, i.e. $D_{m}=I+(1-p) \frac{1}{2} \gamma \hat{a}^{2}$. But then the $\mathrm{E}$ has an incentive to offer the contract set of proposition 7 when he receives the good signal in order to avoid paying the markup in medium states. Thus, the VC will be able to deduce the true state of the project and consequently, the E will also adapt the bad signal contract so that in equilibrium, the contracts of proposition 7 will be offered.

Taking the optimal design of the different support programs derived in subsection 3.3 as given, we know that for investment grants with a liquidation preference (regardless of the type of grant), the VC's claim in the bad state is only guaranteed without exerting any effort if the spill-over effect exceeds the critical level of $S\left(e_{g}^{*}, a_{g}^{*}, \underline{\alpha}_{a_{b}=0}\right)=\frac{(1-p)(1-q)}{p q}[I-l(0)]$. If the spill-over effect is smaller or the $\mathrm{VC}$ does not hold a liquidation preference, the $\mathrm{E}$ does not have an incentive to overstate and thus investment grants only have distributional effects.

Finally, as far as ex ante grants are concerned, the E has an incentive to overstate whenever $E A G+l(0) \geq I$. As can be seen in table 8.3, this is given for $S\left(e_{g}^{*}, a_{g}^{*}, \underline{\alpha}_{a_{b}=0}\right)>$ $\frac{A}{p q}+\left[1+\frac{(1-p)(1-q)}{p q}\right][I-l(0)]$ for a relatively small A while for a relatively large A, $S\left(e_{g}^{*}, a_{g}^{*}, \underline{\alpha}_{a_{b}=0}\right)>\frac{1}{p q}[I-l(0)]$. In this case, the $\mathrm{E}$ will always be able to compensate the $\mathrm{VC}$ in the bad state of the project and so he will offer the same set of contracts as in the case of guarantee programs irrespective of the signal he receives. If the spill-over effect is smaller (just as in the case of investment grants), the E no longer has an incentive to overstate his private information. Thus he will offer the contracts of proposition 7 . 


\section{Summary of the Influence of Public Intervention on}

\section{Contract Design}

To sum up, an overview of the influence of the analyzed public policy programs on contract design for both experienced and inexperienced markets is presented. We distinguish between two cases: the indications without parentheses refer to the case of a large spill-over effect $-S\left(e_{g}^{*}, a_{g}^{*}, \underline{\alpha}\right)>\left[1+\frac{(1-p)(1-q)}{p q}\right][I-l(0)]+\frac{A}{p q}-$ while the indications in parentheses refer to the case of a small spill-over effect: $S\left(e_{g}^{*}, a_{g}^{*}, \underline{\alpha}\right) \leq \frac{(1-p)(1-q)}{p q}[I-l(0)]^{9}$. Moreover, as concerns ex ante grants, we report the results of a relatively small $A^{10}$.

The second column of the table indicates whether the public policy program increases the contracting parties incentives in the good state to public first-best effort levels. The next three columns refer to the case of inexperienced markets. The third column indicates the inherent innovative value of the least innovative project which still gets financed, given the respective policy intervention. In the fourth column, we show the impact of the public policy program on all projects which would also have been financed without intervention - provided that the public institution cannot observe the innovative value of each project $\alpha$ as in our analyses of section 3.3. The last column with respect to inexperienced markets points out the total welfare effect of each public policy measure. Thereby, we make three different assumptions about the observability of the inherent innovative value by the public institution: non-observability as in the analyses of section 3.3; the observability of two groups of projects with an inherent innovative value larger

\footnotetext{
${ }^{9}$ For $\frac{(1-p)(1-q)}{p q}[I-l(0)]<S\left(e_{g}^{*}, a_{g}^{*}, \underline{\alpha}\right) \leq\left[1+\frac{(1-p)(1-q)}{p q}\right][I-l(0)]+\frac{A}{p q}$, the only change - compared to the case of a large spill-over effect - concerns ex-ante grants: the minimum inherent innovative value increases in inexperienced markets and they are no longer welfare-decreasing in experienced markets with a small A.

${ }^{10}$ More concretely for $\mathrm{A}<[(1-\mathrm{p}) \mathrm{q}+(1-\mathrm{q}) \mathrm{p}][\mathrm{I}-\mathrm{l}(0)]$. The results do not change fundamentally, however for a larger A. The only difference is a change in the respective critical values of the spill-over effect.
} 


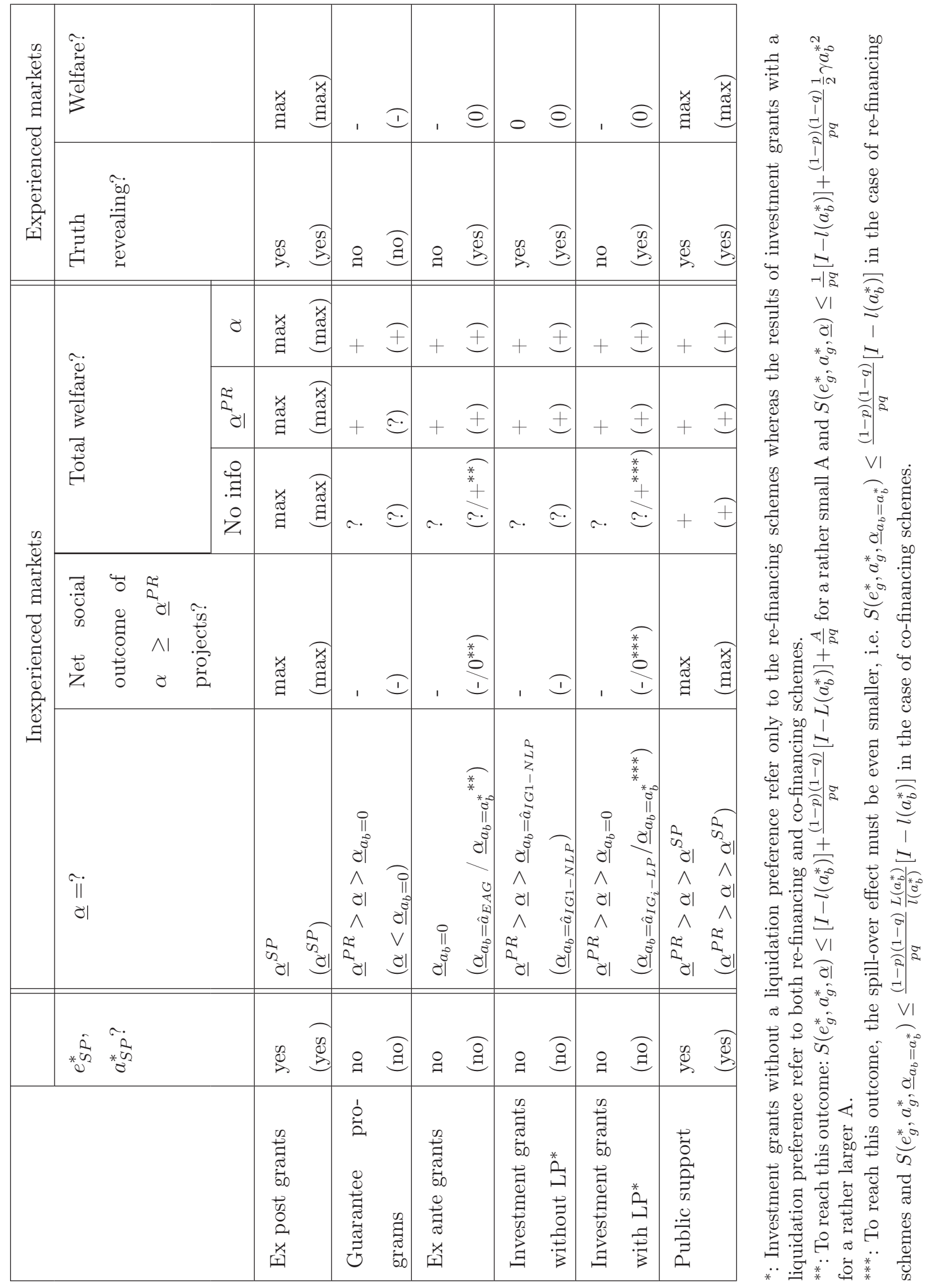


or smaller than $\underline{\alpha}^{P R}$; and the observability of the inherent innovative value $\alpha$ of each project. Under the second assumption, the public institution would not offer support to all those projects which would have been financed without intervention (i.e. all projects with an inherent innovative value of $\alpha \geq \underline{\alpha}^{P R}$ ) and whose outcome is not increased by the intervention. Under assumption three, support can decrease with an increase in the innovative value which, in turn, increases the outcome of these projects because of the limitation of the negative incentive effects ${ }^{11}$.

As we can see in the table, independently of the size of the spill-over effect and the assumption about the observability of $\alpha$, only ex post grants maximize the welfare in both experienced and inexperienced markets but public support maximizes welfare in experienced markets and continues to be welfare-increasing in inexperienced markets. Investment grants, ex ante grants and guarantee programs (with a large spill-over effect) are welfare-increasing in inexperienced markets if the public institution can divide the projects into at least two groups. If this is not the case, their welfare effect will depend on the parameters. However, it is important to emphasize the fact that guarantee programs are always welfare-decreasing in experienced markets whereas ex ante grants and investment grants with a liquidation preference of the $\mathrm{VC}$ are only welfare-decreasing with a large spill-over effect.

\section{Robustness Checks}

We will now introduce an alternative specification of the spill-over effect in order to check our results for robustness. It is clear that a spill-over effect which depends on the effort choices can only be internalized by giving additional incentives to the contracting

\footnotetext{
${ }^{11}$ Note that with this assumption, the above determined optimal support schemes would apply only for the least innovative socially profitable project.
} 
parties. This reduces the set of possible optimal public policy measures. Therefore, we will check our results for a spill-over effect which is independent of the effort levels. Let $S(\alpha)$ be the spill-over effect for a project with $V>I$ and the inherent innovative value $\alpha$. This implies one crucial change with respect to our previous analyses: private firstbest effort levels now correspond to public first-best effort levels in all three states of the project. Thus in inexperienced markets, public intervention can focus on getting all socially profitable projects financed whereas in experienced markets, there is no longer any need for public intervention. Still, our qualitative results do not change considerably with this alternative specification. Ex post grants continue to be welfare-maximizing in both scenarios. Investment grants with a liquidation preference and ex ante grants can maximize social welfare in inexperienced markets with a relatively small spill-over effect and are otherwise welfare-enhancing if the public institution can identify the two groups of projects mentioned. In the latter case, investment grants (with re-financing schemes) without a liquidation preference and guarantee programs with a relatively large spill-over effect are also welfare-enhancing. In experienced markets, however, guarantee programs are still always welfare-decreasing and ex ante grants and investment grants with a liquidation preference continue to be welfare-decreasing with a large spill-over effect. Moreover, it is obvious that public support is no longer an adequate instrument. Thus the introduction of this alternative specification seems to strengthen our results about the robustness and riskiness of the different public policy programs.

A further point that we wish to comment upon is our assumption of risk-neutrality for both contracting parties. The assumption of risk-neutral Es is deemed to be maintainable as the latter do not invest any private wealth in the project. With respect to the VCs, it could be argued that they can be modelled as being risk-neutral because they hold a diversified portfolio of companies. But the question is whether our results are robust for 
contracting with risk-averse VCs. The answer is yes. We wish to illustrate this out by means of guarantee programs, which are often considered as aiming at contracting with risk-averse agents. Without a guarantee program, we are confronted with the traditional trade-off between giving incentives and offering risk insurance. We will never achieve an optimal risk allocation and projects will only get financed if the project is sufficiently innovative so that the risk premium can be paid by the $\mathrm{E}$ in the good state of the project. A guarantee program ensures an optimal risk allocation, and less innovative but socially profitable projects will get financed. The mechanisms, however, do not change in comparison to the case of risk-neutral VCs. The same is true for experienced markets: risk aversion does not have any influence if the truth-revealing condition, which eliminates the project's risk, is achieved.

A final point of the model which could attract criticism is the fact that the doublesided moral hazard problem can be solved by the market due to specific assumptions of the model. One reply might be that the deduced type of contract is empirically well confirmed. Moreover, the aim of the paper was to focus on public policy strategies in order to internalize the spill-over effect related to innovation - a stated purpose in many policy agendas. But what would it mean for our results if the double-sided moral hazard problem were not solved by the market? If the private first-best effort levels were not implemented by the market, the government would have to provide further incentives to increase effort levels. As shown above, this can only be achieved through ex post grants and public support. The other programs would have the same effects on the range of financed projects and the contract mechanisms as described, only the effort levels would be even more suboptimal than in the case of our framework. Thus, the qualitative results concerning the robustness of ex post grants and the riskiness of guarantee programs, ex ante grants and investment grants with a liquidation preference would not change, but 
the advantages of ex post grants would grow as they could also provide these further incentives.

\section{Conclusion}

We have analyzed different public policy programs by placing special emphasis on their impact on contract design. Therefore, we adapted the sequential double-sided moral hazard framework of Schmidt [2003] and the double-sided adverse selection framework of Houben [2002] in order to compare the results of both frameworks and make these models tractable for the purposes of this paper. Furthermore, we allowed for the necessity of public intervention by incorporating a spill-over effect which may be proportional to the outcome of the successful project and can then be influenced by both parties through their effort decisions or which may be independent of it. We consider that the analysis of the impact of public policy programs on contract design is especially important as explicit contracts play a prevalent role in venture capital finance and are quite complex and sensitive.

We have found that program adequacy depends on the size and specification of the spill-over effect and the experience of the VCs and the Es, as only with highly experienced contracting parties is signalling possible. If we are confronted with an inexperienced market and a spill-over effect which is independent of the effort levels, the public first-best situation can be reached by ex post grants and, under some conditions, by ex ante grants and investment grants. The effect of guarantee programs depends on the concrete parameter constellation. If the spill-over effect depends on the effort levels, only ex post grants maximize welfare but ex ante and investment grants as well as guarantee programs with a relatively large spill-over effect continue to be welfare-enhancing if the public institution can divide the projects at least into the two groups mentioned with respect to their inher- 
ent innovative value. Otherwise, their welfare effect depends on the concrete parameters. Finally, public support is always welfare-increasing with a dependent spill-over effect.

If we have an experienced market and an independent spill-over effect, we do not need any public intervention in order to achieve the public first-best situation. However, ex post grants and investment grants (with re-financing schemes) without a liquidation preference by the $\mathrm{VC}$ do not destroy the contract mechanisms and so they have only distributional effects ( this is obviously due to our assumption of lump-sum financing). Guarantee programs, on the contrary, always destroy the truth-revealing mechanism and ex ante grants and investment grants with a liquidation preference by the VC may do this under certain conditions too. If we are confronted with a dependent spill-over effect, only ex post grants and public support are welfare-maximizing. Investment grants (with re-financing schemes) without a liquidation preference have only distributional effects, but guarantee programs and sometimes ex ante grants and investment grants with a liquidation preference by the VC continue to destroy the truth-revealing mechanism.

In conclusion, we have observed that guarantee programs, ex ante grants and investment grants with a liquidation preference are dangerous as they can destroy the contract mechanisms in the case of experienced markets. Public support is only useful with dependent spill-overs. Ex post grants are quite decidedly the most suitable instrument, as they guarantee the first-best situation regardless of the maturity of the market and the specification of the spill-over effect. 


\section{References}

P. Aghion and P. Howitt. A model of growth through creative destruction. Economterica, 60:323-351, 1992.

D. Audretsch and R. Thurik. Linking entrepreneurship to growth. OECD STI Working Paper, 2001.

C. Casamatta. Financing and advising: Optimal financial contracts with venture capitalists. Journal of Finance, 58:2059-2086, 2003.

M. Da Rin, G. Nicodano, and A. Sembenelli. Public policy and the creation of active venture capital markets. Working Paper, 2004.

P. D'Souza. Venture Capital and Asymmetric Information. dissertation.de Berlin, 2001.

EIB. Research, development and innovation. Practical Investor's Guide to EIB Financing, 2004.

Z. Griliches. The search for research and development spillovers. Scandinavian Journal of Economics, 94:S29-47, 1992.

E. Houben. Venture capital, double-sided adverse selection, and double-sided moral hazard. Working Paper, 2002.

S. Kaplan and P. Strömberg. Financial contracting theory meets the real world: An empirical analysis of venture capital contracts. Review of Economic Studies, 70:281$315,2003$.

C. Keuschnigg. Optimal public policy for venture capital backed innovation. 2003. Working Paper Series.

C. Keuschnigg and S.B. Nielsen. Tax policy, venture capital and entrepreneurship. Journal of Public Economics, 87:175-203, 2003.

C. Keuschnigg and S.B. Nielsen. Start-ups, venture capitalists and the capital gains tax. Journal of Public Economics, 88:1011-1042, 2004.

S. Kortum and J. Lerner. Assessing the contribution of venture capital to innovation. Rand Journal, 31:674-692, 2000.

J. Lerner. The government as venture capitalist: The long-run impact of the sbir program. Journal of Business, 72:285-318, 1999.

J. Lerner. When bureaucrats meet entrepreneurs: The design of effective public venture capital programmes. Economic Journal, 112:F73-F84, 2002.

OECD. Science, Technology and Industry Outlook: Drivers of Growth: Information Technology, Innovation and Entrepreneurship. OECD, 2001. 
R.G. Rajan and L. Zingales. Financial systems, industrial structure, and growth. Oxford Review of Economic Policy, 17:467-482, 2001.

R. Repullo and J. Suarez. Venture capital finance: A security design approach. Review of Finance, 8:75-108, 2004.

P.M. Romer. Increasing returns and long run growth. Journal of Political Economy, 94: 1002-1037, 1986.

K.M. Schmidt. Convertible securities and venture capital finance. Journal of Finance, 58:1139-1166, 2003.

T. Tykvova. What do economists tell us about venture capital contracts? 2000. Discussion Paper No. 00-62. 


\section{Appendix}

\subsection{Proof of Proposition 1:}

The E will offer the contract which yields him the highest profit in $t_{5}$. Thereby, his profit depends on the effort levels chosen by both contracting parties as well as the amount to be paid to the VC. In subsection 3.1, we have shown that the project's surplus is maximized when both contracting parties choose private first-best effort levels. Now we will show that neither equity nor debt nor debt-equity mixes give both contracting parties simultaneously an incentive to choose private first-best effort levels in all states of the project, while preferred stock contracts are able to do so. We assume for simplicity's sake that the initial contract cannot be renegotiated at any point in the relationship. In section III, however, we will derive sufficient conditions to guarantee that the deduced convertible preferred stock contracts are renegotiation-proof. Finally, as a transfer of control rights cannot convey any additional information, we assume that the E will hold them and thus will obtain private benefits regardless of the type of contract chosen ${ }^{12}$.

\section{Pure Equity, Pure Debt and Debt-Equity Mixes \\ 1. Pure Equity:}

The E offers the VC an equity stake $s^{e q} \in(0,1]$. Payoff functions of both contracting parties for the different states are given by

$$
\begin{aligned}
\Pi^{E}= & \left(1-s^{e q}\right) \bar{x}(e, a, \alpha)-\frac{1}{2} \beta e^{2}+B & & \text { for } \theta=\text { good } \\
& \left(1-s^{e q}\right) I-\frac{1}{2} \beta e^{2}+B & & \text { for } \theta=\text { medium } \\
& \left(1-s^{e q}\right) l(a)-\frac{1}{2} \beta e^{2}+B & & \text { for } \theta=\text { bad }
\end{aligned}
$$

\footnotetext{
${ }^{12}$ This proof is based on the insights of Schmidt [2003].
} 


$$
\begin{aligned}
\Pi^{V C}= & s^{e q} \bar{x}(e, a, \alpha)-\frac{1}{2} \gamma a^{2} & \text { for } \theta=\operatorname{good} \\
& s^{e q} I-\frac{1}{2} \gamma a^{2} & \text { for } \theta=\text { medium } \\
& s^{e q} l(a)-\frac{1}{2} \gamma a^{2} & \text { for } \theta=\text { bad }
\end{aligned}
$$

Both contracting parties will choose their effort levels so as to maximize their payoffs.

Effort levels are thus given by the FOCs:

$$
\begin{aligned}
e_{g}^{e q} & =\left(1-s^{e q}\right) \frac{\partial \bar{x}(e, a, \alpha)}{\partial e}<e_{g}^{*} \\
e_{m}^{e q} & =0=e_{m}^{*} \\
e_{b}^{e q} & =0=e_{b}^{*} \\
a_{g}^{e q} & =s^{e q} \frac{\partial \bar{x}(e, a, \alpha)}{\partial a} \leq a_{g}^{*} \\
a_{m}^{e q} & =0=a_{m}^{*} \\
a_{b}^{e q} & =s^{e q} \frac{\partial l(a)}{\partial a} \leq a_{b}^{*}
\end{aligned}
$$

\section{Pure Debt:}

The E offers the VC a debt claim $D>I$ (due to the the VC's participation constraint).

Payoff functions of both contracting parties for the different states are given by

$$
\begin{array}{rlrl}
\Pi^{E}= & \bar{x}(e, a, \alpha)-\frac{1}{2} \beta e^{2}+B-\max [D, \bar{x}(e, a, \alpha)] & & \text { for } \theta=\operatorname{good} \\
& B-\frac{1}{2} \beta e^{2} & & \text { for } \theta=\text { medium } \\
& B-\frac{1}{2} \beta e^{2} & & \text { for } \theta=\text { bad } \\
\Pi^{V C}= & \max [D, \bar{x}(e, a, \alpha)]-\frac{1}{2} \gamma a^{2} & \text { for } \theta=\operatorname{good} \\
& I-\frac{1}{2} \gamma a^{2} & \text { for } \theta=\operatorname{medium} \\
& l(a)-\frac{1}{2} \gamma a^{2} & & \text { for } \theta=\text { bad }
\end{array}
$$


Both contracting parties will choose their effort levels so as to maximize their payoffs. Thus effort levels are again given by the FOCs in bad and medium states.

$$
\begin{aligned}
& e_{m}^{e q}=0=e_{m}^{*} \\
& e_{b}^{e q}=0=e_{b}^{*} \\
& a_{m}^{e q}=0=a_{m}^{*} \\
& a_{b}^{e q}=\frac{\partial l(a)}{\partial a}=a_{b}^{*}
\end{aligned}
$$

In the good state, however, we have to distinguish between two cases:

Case 1: $D<\bar{x}\left(e_{g}^{*}, \delta, \alpha\right)-\frac{1}{2} \gamma e_{g}^{* 2}$ : The VC will choose a marginal effort level in order to guarantee her claim and the $\mathrm{E}$ is full residual claimant and will therefore choose $e=e_{g}^{*}$. Case 2: $D>\bar{x}\left(e_{g}^{*}, \delta, \alpha\right)-\frac{1}{2} \gamma e_{g}^{* 2}:$ If $e>0$, the VC will choose an effort level of $a_{\min }^{g} \in\left[\delta, a_{g}^{*}\right]$ which is necessary to guarantee her claim $\mathrm{D}$ or at least maximize her payoff. If $e=0$, due to the imperfect substitutability, the VC will also choose an effort level of 0 . The E anticipates that he will never make positive monetary profits for all $e>0$ and will therefore always choose an effort level of 0 .

\section{Debt-Equity mixes:}

Consider any debt-equity contract $\left(D^{e q, D}, s^{e q, D}\right)$. Deriving the FOCs analogous to the cases of pure equity and pure debt financing yields at least suboptimal effort levels of both contracting parties in the good state as the marginal returns on the investments to the $\mathrm{E}$ and the $\mathrm{VC}$ are still smaller than one. In the bad state of the project, the $\mathrm{VC}$ invests efficiently only if $D^{e q, D}>l\left(a_{b}^{*}\right)$.

To sum up, we have shown that none of the contracts can implement private first-best effort levels of both contracting parties in all states of the project. 


\section{Convertible Preferred Stock Contracts}

Consider a convertible preferred stock contract with a limited dividend $D$ and a conversion option in an equity stake $s_{1}^{C V}$ in $t_{4}$ if the threshold $z$ is reached. Below, we will derive the optimal design of such a contract. The solution is by backward induction.

\section{The Choice of Effort Levels:}

1. Bad State: If $D \geq l\left(a_{b}^{*}\right)$, the VC gets the total surplus from the project and so has an incentive to invest efficiently. If $l(0)<D<l\left(a_{b}^{*}\right)$, the $\mathrm{VC}$ will choose the effort level $a_{b}^{d e v \cdot}$ such that the following condition holds: $l\left(a_{b}^{d e v \cdot}\right)=D$. If $D \leq l(0)$ the VC's claim is guaranteed independently of her effort effort level and she will choose $\mathrm{a}=0$. The $\mathrm{E}$ knows that he cannot influence the outcome and so chooses an effort level of 0 .

2. Medium State: The VC and the E know that the project's surplus is independent of the effort levels and so both choose an effort level of 0 .

3. Good State: The contract includes a conversion option in $t_{4}$ which is linked to the threshold z. This threshold indicates the minimum surplus which must be reached for conversion to be possible. Suppose that the threshold is set at $z=\bar{x}(\underline{e}, \underline{a}, \alpha)$. The solution is by backward induction. We will derive the conditions which have to apply in order to guarantee that both contracting parties choose $\underline{a}$ and $\underline{e}$ respectively.

a) Choice of effort level by the VC:

Case 1: $\mathrm{e}=\underline{\mathrm{e}}$ : The $\mathrm{VC}$ anticipates that the threshold $\mathrm{z}$ will only be reached if she exerts an effort level of $a \geq \underline{a}$. After conversion, the VC's payoff amounts to

$$
\Pi_{g}^{V C}=s_{1}^{C V} \bar{x}(\underline{e}, a, \alpha)-\frac{1}{2} \gamma a^{2}
$$

We know that the VC would maximize her profit by choosing

$$
\hat{a}_{g}^{C V}=\arg \max \left[s_{1}^{C V} \bar{x}(\underline{e}, a, \alpha)-\frac{1}{2} \gamma a^{2}\right]
$$




$$
\hat{a}_{g}^{C V}<\underline{a}
$$

holds, the threshold is not reached with $\hat{a}_{g}^{C V}$ and as the VC's profit is decreasing in $a$ for $a>\hat{a}_{g}^{C V}$, the VC will choose $a=\underline{a}$ whenever conversion pays off for her ${ }^{13}$. This is the case when the VC's profit with conversion amounts at least to the profit without conversion which depends on the size of claim $D$.

1. Assume that $D \leq I$. In this case, the $\mathrm{VC}$ can guarantee herself the payoff without exerting any effort. Conversion is only profitable if

$$
I C(V C)_{c o n v .}^{1}: \quad s_{1}^{C V} \bar{x}(\underline{e}, \underline{a}, \alpha)-\frac{1}{2} \gamma \underline{a}^{2} \geq D
$$

2. Assume that $D>I$, then the $\mathrm{VC}$ will choose an effort level of $a_{\min }^{g} \in\left[\delta, a_{g}^{*}\right]$ which is necessary to guarantee her claim D or at least minimize her loss. Conversion is profitable if

$$
\begin{aligned}
I C(V C)_{\text {conv. }}^{2}: \quad s_{1}^{C V} \bar{x}(\underline{e}, \underline{a}, \alpha)-\frac{1}{2} \gamma \underline{a}^{2} \geq & \\
& \min \left[D, x\left(\underline{e}, a_{b}^{*}, \alpha\right)\right]-\frac{1}{2} \gamma\left(a_{g}^{\text {min }}\right)^{2}
\end{aligned}
$$

Case 2: $0<e<\underline{e}:^{14}$ The VC anticipates that the threshold $\mathrm{z}$ will only be reached if she exerts $a=a_{\text {conv }}^{\text {min }}>\underline{a}$. As the VC's payoff with conversion is decreasing in $a$ for $a>\underline{a}$ (see equation 42), the $\mathrm{VC}$ will choose the smallest $a_{\text {conv }}^{\min }$ for which conversion is still possible (i.e. $\bar{x}\left(e, a_{c o n v}^{\min }, \alpha\right)=z$ ) provided that conversion still pays off for her. Without conversion, the $\mathrm{VC}$ will have to choose $a_{g}^{\min }$ in order to guarantee her claim

\footnotetext{
${ }^{13} \mathrm{As} \hat{a}_{g}^{C V}<a_{g}^{*}$ and our aim is to show that private first-best effort levels of both contracting parties can be implemented through an adequately designed conversion option, below, we will restrict ourselves to this case. But we will have to recheck the limiting assumption in a final step.

${ }^{14}$ For $e=0$, conversion is never possible due to the limited substitutability assumption.
} 
$D$. In order to ensure that conversion is not profitable for the VC in this case, the following condition must apply:

$$
s_{1}^{C V} \bar{x}(\underline{e}, \underline{a}, \alpha)-\frac{1}{2} \gamma a_{c o n v}^{\min 2} \leq D-\frac{1}{2} \gamma\left(a_{g}^{\min }\right)^{2}
$$

Moreover, we know that if the VC cannot guarantee her claim $D$ by choosing $a_{g}^{*}$, she will not further increase her effort level as her net payoff would decrease. In this case, the following condition must hold in order to guarantee that conversion does not pay off for the VC:

$$
s_{1}^{C V} \bar{x}(\underline{e}, \underline{a}, \alpha)-\frac{1}{2} \gamma a_{c o n v}^{\min 2} \leq x\left(\hat{e}, a_{g}^{*}, \alpha\right)-\frac{1}{2} \gamma a_{g}^{* 2}
$$

But as $x\left(\hat{e}, a_{g}^{*}, \alpha\right)-\frac{1}{2} \gamma a_{g}^{* 2}>D-\frac{1}{2} \gamma\left(a_{g}^{m i n}\right)^{2}$ with $a_{g}^{m i n}>a_{b}^{*}$, condition 46 is automatically fulfilled if condition 45 applies. Furthermore, if

$$
\frac{1}{2} \beta \underline{e}^{2}-\frac{B}{p q} \geq \bar{x}(\underline{e}, \underline{a}, \alpha)-\frac{1}{2} \gamma \underline{a}^{2}-\bar{x}(\underline{e}, \delta, \alpha)
$$

the VC needs only to choose a marginal effort level to guarantee her claim $D$ (for all privately profitable projects) provided that the E chooses his effort level $\underline{e}$. Consequently, due to the concavity of $x$ in $a$, a deviation of the $\mathrm{E}$ is always more costly for the $\mathrm{VC}$ with respect to effort costs when converting than without converting. Thus 45 is always fulfilled if $s_{1}^{C V} \bar{x}(\underline{e}, \underline{a}, \alpha)-\frac{1}{2} \gamma \underline{a}^{2} \leq D$. We will refer to this condition as the E's non-deviation condition. If our assumption did not hold, a convertible security which implements $\underline{e}$ and $\underline{a}$ could only be implemented for more innovative projects for which $D$ is guaranteed with $a=\delta$ only. Less innovative projects may be partially financed anyhow - the security depending on the parameters - but as private first-best effort levels of both contracting parties could never be achieved, the range of privately profitable projects would be reduced.

Case 3: $e>\underline{e}$ : In order to be able to exert her conversion option, the $\mathrm{VC}$ must invest 
only $\underline{\hat{a}}<\underline{a}$ with $\bar{x}(e, \underline{\hat{a}}, \alpha)=z$. The incentive constraints of case 1 which guarantee conversion to be profitable change accordingly.

b) Choice of effort level by the E:

Given the conditions derived in item a), conversion is only implementable for $e \geq \underline{e}$. As the $\mathrm{E}$ anticipates that his profit after conversion continues to be $\left(1-s_{1}^{C V}\right) z$ even if he exerts an effort level of $e>\underline{e}$, he will choose $e=\underline{e}$ whenever conversion is worthwhile for him. This is the case if the following condition applies:

$$
I C(E)_{\text {conv. }}^{i}: \quad\left(1-s_{1}^{C V}\right) \bar{x}(\underline{e}, \underline{a}, \alpha)-\frac{1}{2} \beta \underline{e}^{2}+B \geq \Pi(E)_{d e v}^{i}
$$

with $\Pi_{\text {dev }}^{i}$ depending on the size of the claim $\mathrm{D}$ :

Case 1: If $D \leq I$, the E anticipates that the $\mathrm{VC}$ will choose an effort level of 0 and because of the limited substitutability, he will also choose an effort level of 0 . Then, in case of deviation, the payoff in the good state amounts to $\Pi(E)_{d e v}^{1}=I-D+B$. Case 2: If $I<D<\bar{x}(\underline{e}-\delta, \delta, \alpha)-\frac{1}{2} \beta(\underline{e}-\delta)^{2}$, the $\mathrm{E}$ anticipates that he will be full residual claimant and will choose an effort level of $\underline{e}-\delta$. Then, the profit with deviation amounts to:

$\Pi(E)_{d e v}^{2}=\bar{x}(\underline{e}-\delta, \delta, \alpha)-\frac{1}{2} \beta(\underline{e}-\delta)^{2}-D+B$

Case 3: If $D>\bar{x}(\underline{e}-\delta, \delta, \alpha)-\frac{1}{2} \beta(\underline{e}-\delta)^{2}$, the $\mathrm{E}$ will never make positive profits regardless of his effort level and will choose an effort level of 0 . In this case $\Pi(E)_{d e v}^{3}=$ $B$.

\section{The Contract Design:}

In a first step, we will restrict ourselves to contracts for which the $\mathrm{PC}(\mathrm{VC})$ is binding. If such a contract exists which induces private first-best effort levels of both contracting parties, we know that no alternative contract exists which increases the E's profit ${ }^{15}$.

\footnotetext{
${ }^{15}$ If such a contract does not exist, we will have to look also at convertible preferred stock contracts for
} 
We know from the analysis of the choice of effort levels that in order to guarantee an efficient effort choice of the $\mathrm{VC}$ in the bad state of the project $D \geq l\left(a_{b}^{*}\right)$. The $\mathrm{E}$ has an incentive to minimize the loss in the bad state because this reduces the amount to be paid to the $\mathrm{VC}$ in the good state and thus maximizes his profit. Thus we want this condition to hold. Then, the $\mathrm{PC}(\mathrm{VC})$ is given by:

$$
(1-p)(1-q)\left[l\left(a_{b}^{*}\right)-\frac{1}{2} \gamma a_{b}^{* 2}\right]+[(1-p) q+(1-q) p] \min [I, D]+p q\left[s_{1}^{C V} \bar{x}(\underline{e}, \underline{a}, \alpha)-\frac{1}{2} \gamma \underline{a^{2}}\right] \geq I
$$

If the $\mathrm{PC}(\mathrm{VC})$ is binding, we arrive at the following conversion stake $s_{1}^{C V}$ :

$$
\begin{aligned}
s_{1}^{C V} & =\frac{C+\frac{1}{2} \gamma \underline{a}^{2}}{\bar{x}(\underline{e}, \underline{a}, \alpha)} \\
& \text { with } C=I+\frac{(1-p)(1-q)}{p q}\left[I-L\left(a_{b}^{*}\right)\right]+\frac{(1-p) q+(1-q) p}{p q}[I-\min [I, D]]
\end{aligned}
$$

Moreover, we know that the incentive constraints mentioned, which guarantee that conversion is profitable for both contracting parties, must hold. As (44) is automatically fulfilled when (43) holds, we will only take into account the latter in order to avoid case differentiation below. As far as the $\mathrm{E}$ is concerned, if (48) holds for $i=2$, then it is automatically fulfilled for $i=1,3$ and thus again, we can restrict ourselves to the case of $i=2$. This means that the deduced contracts may not be the only possible equilibrium contracts. But as our aim is to analyze public intervention on contract design and our results do not change, we will use this procedure for the sake of simplicity. Including $s_{1}^{C V}$ in the E's maximization problem and anticipating that the limited liability constraint of the $\mathrm{E}$ is always binding in the bad state (as shown, the $\mathrm{E}$ will choose $D \geq l\left(a_{b}^{*}\right)$ in order to minimize the loss) and may be binding in the medium state yields the following which the $\mathrm{PC}(\mathrm{VC})$ is not binding. In this case, however, the convertible preferred stock contract needs not to be the profit-maximizing contract. 
maximization problem:

$$
\begin{aligned}
\max _{\underline{e}, \underline{a}} \Pi_{C V}^{E}=p q\left[\bar{x}(\underline{e}, \underline{a}, \alpha)-\frac{1}{2} \beta \underline{e}^{2}-\frac{1}{2} \gamma \underline{a}^{2}-C\right]+[(1-p) q+(1-q) p][I-\min [I, D]]+B \\
\text { s.t. } \\
L L C(E)_{g} \\
P C(E): \quad \Pi_{C V}^{E} \geq 0 \\
I C(V C)_{\text {conv. }}: \quad C \geq D \\
I C(E)_{\text {no-dev. }}: \quad C \leq D \\
I C(E)_{\text {conv. }}: \quad \quad \bar{x}(\underline{e}, \underline{a}, \alpha)-\frac{1}{2} \beta \underline{e}^{2}-\frac{1}{2} \gamma \underline{a}^{2}-C \geq \bar{x}(\underline{e}-\delta, \delta, \alpha)-\frac{1}{2} \beta(\underline{e}-\delta)^{2}-D
\end{aligned}
$$

As far as optimal effort levels are concerned, no further restrictions need to be considered and the FOCs yield the following optimal effort levels:

$$
\begin{aligned}
\underline{e}^{*} & =\frac{1}{\beta} \frac{\partial \bar{x}(\underline{e}, \underline{a}, \alpha)}{\partial \underline{e}}=e_{g}^{*} \\
\underline{a}^{*} & =\frac{1}{\gamma} \frac{\partial \bar{x}(\underline{e}, \underline{a}, \alpha)}{\partial \underline{a}}=a_{g}^{*}
\end{aligned}
$$

This implies that the E introduces the threshold $z=\bar{x}\left(e_{g}^{*}, a_{g}^{*}, \alpha\right)$ in order to induce both parties to choose private first-best effort levels. In addition, our limiting assumption (42) is also confirmed as indeed is $\hat{a}_{g}^{C V}<a_{g}^{*}$.

With regard to the conditions, we know that for private first-best effort levels, equation 47 is fulfilled and that the $I C(E)_{n o-d e v}$. demands $D \geq C$. In addition, the $I C(V C)_{\text {conv }}$. and the $I C(E)_{c o n v}$. must also be fulfilled. It is obvious that all conditions hold for $D=C$. Thus $C=D=I+\frac{(1-p)(1-q)}{p q}\left[I-L\left(a_{b}^{*}\right)\right]$.

The E's profit is thus maximized with this contract and amounts to:

$$
\Pi_{C V}^{E}=p q\left[\bar{x}\left(e_{g}^{*}, a_{g}^{*}, \alpha\right)-\frac{1}{2} \beta e_{g}^{* 2}-\frac{1}{2} \gamma a_{g}^{* 2}-C\right]+B
$$

As $B \leq p q \frac{1}{2} \beta e_{g}^{* 2}$, the $L L C(E)_{g}$ is automatically satisfied when $\mathrm{PC}(\mathrm{E})$ holds. And the $\mathrm{PC}(\mathrm{E})$ is fulfilled for all privately profitable projects. 


\section{A Note on the Renegotiation Issue}

Schmidt [2003] shows that if renegotiation is possible, the bargaining power of the E and/or the benefit from renegotiation must be sufficiently small to ensure that the convertible security does implement the first-best situation. Below, we will look at the impact of possible renegotiation within our modified framework. We know that the E has an incentive to choose his private first-best effort level in the good state only because of the sequential decision structure, which implies a threat by the VC not to convert and not to exert effort if $e<e_{g}^{*}$. In this case, however, scope for renegotiation exists in $t=2,5$ : the E could adapt the threshold $\mathrm{z}$ and the equity stake $s_{1}^{C V}$ in order to make it profitable for the $\mathrm{VC}$ to convert and to exert her private first-best effort level even though the $\mathrm{E}$ has chosen $e<e_{g}^{*}$. Let us assume that renegotiation takes place whenever there is scope for an efficiency improvement and that the $\mathrm{E}$ and the $\mathrm{VC}$ split the surplus from renegotiation proportionately $(\lambda, 1-\lambda$, ) where $\lambda \in[0,1]$ is the fraction that goes to the E.

If the deviation of the $\mathrm{E}$ is relatively small and the $\mathrm{VC}$ can still guarantee her claim D without exerting any effort (or only the marginal effort level $\delta$ ), it is obvious that, in our case with $\mathrm{D}=\mathrm{C}$, renegotiation never pays off for the E. While with conversion, he has to pay $C+\frac{1}{2} \gamma a_{g}^{* 2}$ to the $\mathrm{VC}$, with deviation and renegotiation, he has to pay at least $D+\frac{1}{2} \gamma a_{g}^{* 2}$ and the total surplus of the project is smaller.

However, if the deviation of the $\mathrm{E}$ is relatively large, the $\mathrm{VC}$ will have to choose a higher effort level $a \in\left(0, a_{g}^{*}\right]$ in order to guarantee her claim $D$. If her claim is never guaranteed, she will choose $a_{g}^{*}$ in order minimize her loss - in this case renegotiation is never possible, however. Therefore, with a relatively large deviation, the E's profit after renegotiation amounts to

$$
\Pi_{R}^{E}=-\frac{1}{2} \beta e_{\text {dev. }}^{2}+\lambda\left[\bar{x}\left(e_{\text {dev. }}, a_{g}^{*}, \alpha\right)-\frac{1}{2} \gamma a_{g}^{* 2}-D+\frac{1}{2} \gamma{a_{\text {min }}^{g}}^{2}\right]+B
$$


with $D=\bar{x}\left(e_{\text {dev. }}, a_{\text {min }}^{g}, \alpha\right)$. The last large expression is the smaller, the smaller the effort level chosen by the E. But at the same time, the effort costs of the E are smaller, too. The E will choose the effort level which maximizes his payoff after renegotiation and which amounts to

$$
\hat{e}^{R}=\frac{\lambda}{\beta}\left[\frac{\partial x\left(e_{\text {dev. }}, a_{\min }^{g}, \alpha\right)}{\partial a_{\min }^{g}}+\gamma a_{\min }^{g} \frac{\partial a_{\min }^{g}}{\partial e}\right]
$$

if $\hat{e}^{R} \in\left(0, e_{g}^{*}\right)$ and otherwise to 0 or $e_{g}^{*}-\delta$ respectively. Thus to implement renegotiation proofness for our case with $D=C=\bar{x}\left(e^{R}, a_{\text {min }}^{g}, \alpha\right)$, the E's bargaining power must be sufficiently small, i.e.

$$
\lambda \leq \frac{\bar{x}\left(e_{g}^{*}, a_{g}^{*}, \alpha\right)-\frac{1}{2} \beta e_{g}^{* 2}-\frac{1}{2} \gamma a_{g}^{* 2}-C+\frac{1}{2} \beta e^{R^{2}}}{\left.\bar{x}\left(e^{R}, a_{g}^{*}, \alpha\right)-\frac{1}{2} \gamma a_{g}^{* 2}-D+\frac{1}{2} \gamma{a_{\text {min }}^{g}}^{2}\right]}
$$

We assume this to hold in what follows. Then our derived contract with $D=C$ is renegotiation-proof. Furthermore, we assume that the convertible security contracts continue to be renegotiation-proof in equilibrium with public intervention. This is always the case with guarantee programs and given our assumption also with ex ante grants and investment grants with re-financing. With public support, ex post grants and investment grants with co-financing, however, additional constraints must also be fulfilled ${ }^{16}$ which we assume to hold in the following.

\subsection{Proof of Proposition 7:}

The E will offer the contracts which yield him the highest profit in $t_{5}$. Thereby, his profit depends on three factors: the achievement of truth-revealing by the contracting parties, the effort levels chosen by the contracting parties and the amount paid to the VC. In

\footnotetext{
${ }^{16}$ i.e. $\lambda \leq \frac{(1+\varepsilon) \bar{x}\left(e_{g}^{* S P}, a_{g}^{* S P}, \alpha\right)-\frac{1}{2} \beta e_{g}^{* S P^{2}}-\frac{1}{2} \gamma a_{g}^{* S P^{2}}-D+\frac{1}{2} \beta e_{E P G}^{R}{ }^{2}}{(1+\varepsilon) \bar{x}\left(e_{E P G}^{R}, a_{g}^{* S P}, \alpha\right)-\frac{1}{2} \gamma a_{g}^{* S P} P^{2}-D+\frac{1}{2} \gamma a_{\min }^{g}{ }^{2}}$ for public support and ex post grants, and $\lambda \leq \frac{\bar{x}\left(e_{g}^{*}, a_{g}^{*}, \alpha\right)-\frac{1}{2} \beta e_{g}^{* 2}-\frac{1}{2} \gamma a_{g}^{* 2}-D+\frac{1}{2} \beta e_{I G-C F}^{R}{ }^{2}}{\left.\bar{x}\left(e_{I G-C F}^{R}, a_{g}^{*}, \alpha\right)-\frac{1}{2} \gamma a_{g}^{* 2}-\min \left[D ; x\left(e_{I G-C F}^{R}, a^{\max }, \alpha\right)\right]+\frac{1}{2} \gamma a_{\min }^{g}{ }^{2}\right]}$ with $a^{\max }=\frac{(1-\tau)}{\gamma} \frac{\partial x\left(e_{I G-C F}^{R}, a, \alpha\right)}{\partial a}$ for investment grants with co-financing, provided that effort cost compensation after renegotiation goes only to the VC.
} 
proof 1 , we showed that only convertible preferred stock contracts are able to induce both contracting parties to choose private first-best effort levels in the good state. We will now anticipate this result and therefore restrict to convertible preferred stock contracts in the good state. But we know that in order to induce private first-best effort levels, it is still crucial to achieve truth-revealing ${ }^{17}$. Thus in what follows, we will show that there exist contract sets of preferred stock contracts for which truth-revealing is achieved. Note that for the sake of simplification, renegotiation is excluded at any point of the relationship in our basic analysis, but its influence is studied separately afterwards ${ }^{18}$.

We will first look at the case where the E receives the good signal $\bar{\lambda}$. This means that the project can either be in the good or medium state. As the E aims to induce truthrevealing by the $\mathrm{VC}$, he has to design two different contracts which ensure that the $\mathrm{VC}$ will choose the good state contract only if she receives the good signal and the medium state contract only if she receives the bad signal. Analogous to proof 1 , we again assume that the E will offer a convertible security as good state contract which can only be converted if the threshold $z=\bar{x}(\underline{e}, \underline{a}, \alpha)$ is reached. As far as the medium-state contract is concerned, we know that effort levels do not have any influence on the outcome in this case and the security choice is therefore irrelevant. In the interest of simplicity, we assume that the $\mathrm{E}$ will choose the simplest contract, viz. a contract which implies a fixed payment $D_{m}$. Thus, by additionally including the constraints which must be fulfilled to ensure truth-revealing by the $\mathrm{VC}$ (SSCs), we can state the following modified maximization problem of the $\mathrm{E}$ :

\footnotetext{
${ }^{17}$ If truth-revealing is not achieved, the contracting parties do not know the true state of the project when choosing their effort levels. But even if the true state of the project was common knowledge before choosing the effort levels, the E would have an incentive to induce truth-revealing in order to get all medium state projects financed.

${ }^{18}$ This proof is based on the insights of Houben [2002].
} 


$$
\begin{aligned}
\max \Pi_{C V}^{E}= & q\left[\left(1-s_{2}^{C V}\right) \bar{x}(\underline{e}, \underline{a}, \alpha)-\frac{1}{2} \beta \underline{e}^{2}\right]+(1-q)\left[I-D_{m}\right] \\
\text { s.t. } & \\
P C(E): & \Pi_{C V}^{E} \geq 0 \\
L L C(E)^{g}: & s_{2}^{C V} \leq 1 \\
L L C(E)^{m}: & D_{m} \leq I \\
P C(V C)_{g}: & s_{2}^{C V} \bar{x}(\underline{e}, \underline{a}, \alpha)-\frac{1}{2} \gamma \underline{a}^{2} \geq I \\
P C(V C)_{m}: & D_{m} \geq I \\
S S C(V C)_{g}: & s_{2}^{C V} \bar{x}(\underline{e}, \underline{a}, \alpha)-\frac{1}{2} \gamma \underline{a}^{2} \geq D_{m} \\
S S C(V C)_{m}: & D_{m} \geq D_{g} \\
I C(V C)_{c o n v}: & s_{2}^{C V} \bar{x}(\underline{e}, \underline{a}, \alpha)-\frac{1}{2} \gamma \underline{a}^{2} \geq D_{g} \\
I C(E)_{c o n v}: & \left(1-s_{2}^{C V}\right) \bar{x}(\underline{e}, \underline{a}, \alpha)-\frac{1}{2} \beta \underline{e}^{2} \geq I-D_{g} \\
I C(E)_{n o-d e v}: & s_{2}^{C V} \bar{x}(\underline{e}, \underline{a}, \alpha)-\frac{1}{2} \gamma \underline{a}^{2} \leq D_{g}
\end{aligned}
$$

Analogous to proof 1, we again restrict our analysis to contracts for which the PC(VC)

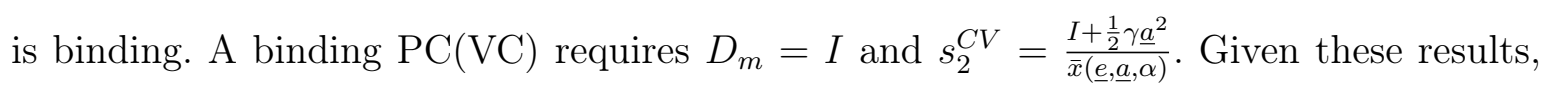
the $S S C(V C)_{g}$, the $\operatorname{LLC}(\mathrm{E}) \mathrm{s}$ as well as the $\mathrm{PC}(\mathrm{E})$ are always fulfilled; the $S S C(V C)_{m}$, the $I C(V C)_{c o n v}$ require that $D_{g} \leq I$; and, at the same time, the $I C(E)_{c o n v}$ demands that $D_{g}>\max \left[0, D_{g}(\min )\right]$ with $D_{g}(\min )=I-\left[\bar{x}(\underline{e}, \underline{a}, \alpha)-\frac{1}{2} \beta \underline{e}^{2}-\frac{1}{2} \underline{a}^{2}-I\right]$ and the $I C(E)_{n o-d e v}$ requires that $D_{g} \geq I^{19}$. We can see that all conditions are simultaneously fulfilled for $D_{g}=I$. Thus, truth-revealing is achieved and analogous to proof 1 private first-best effort levels are implemented.

We will now look at the case where the E receives the bad signal $\underline{\lambda}$. This means that

\footnotetext{
${ }^{19}$ In addition, condition 47 is always fulfilled.
} 
the project can be either in the medium or the bad state. As shown, in the medium state, the security choice is irrelevant and therefore, we assume again that the E offers a simple fixed payment contract for which the $P C(V C)_{m}$ is binding: $D_{m}=I$. If the $\mathrm{VC}$ receives $\underline{\eta}$, she will not accept the offer as her $\mathrm{PC}$ is not fulfilled, whereas if she receives $\bar{\eta}$, her participation constraint is fulfilled and she will accept the offer. Her private information is revealed truthfully.

In a last step, it is important to check that the $\mathrm{E}$ does not have an incentive to deviate, i.e. to offer the contract set related to the good signal if he receives the bad signal and vice-versa. We know that it is efficient to give the $\mathrm{E}$ the control rights in at least one period. Therefore, we assume that with truthful revelation the E receives private benefits in both medium and good states. It is easy to see that the $\mathrm{E}$ does not have an incentive to understate because medium state projects would not get financed and he would lose his private benefits. The overstatement case is represented in the following table:

\begin{tabular}{|c|c|c|}
\hline state & with truthful revelation & with overstatement \\
\hline medium & $B$ & $(B)$ \\
\hline bad & 0 & $(B)$ \\
\hline
\end{tabular}

As can be seen in the table, whether overstatement pays off for the E or not, crucially depends on the control allocation. Convertible preferred stock contracts afford us the possibility of a contingent control allocation: control rights can be given to the $\mathrm{VC}$ with the preferred stock contract, but the E can get them back with conversion which is only possible if the strike $z$ is reached. This can, in turn, only be achieved for good state projects. A similar mechanism is also implementable for preferred stock contracts with a limited dividend which may transfer the control rights to the VC. Here, the E can get back control rights by redeeming the preferred stock contract at a redemption price which must compensate the $\mathrm{VC}$ for his forgone limited dividend $D_{m}$ (thus $P_{m}=I$ ). However, 
as the $\mathrm{E}$ is wealth-constrained, he will be able to redeem the preferred stock at a price $P_{m}=I$ only if the project is at least in the medium state. We see from the table that the E must never hold control rights in both states with overstatement. Thus control rights have to be transferred to the $\mathrm{VC}$ in at least one state giving the $\mathrm{E}$ the option to get them back with truthful revelation. If the E transfers the control rights in the good state but not in the medium state, truth-revealing can only be achieved for $q>0,5$. If the $\mathrm{E}$ transfers control rights in the medium state but not in the good state, truth-revealing by the $\mathrm{E}$ is always guaranteed.

As far as renegotiation is concerned, Houben (2002) shows that the contracts must be slightly modified in the good and medium states in order to make them renegotiationproof. There are two possible cases for profitable renegotiation. First, renegotiation is profitable if the $\mathrm{VC}$ prevents a control transfer back to the E by adopting a certain behavior. Second, renegotiation is profitable if the E deviates in the good state by choosing a lower effort level analogous to proof 1 . As regards the first case, we know that the VC may be interested in deviating because he expects to get a share $(1-\lambda)$ of the renegotiation surplus. One necessary condition for renegotiation is that the E holds sufficient cash flow to compensate the VC. In our framework, this is only given in the good state. Therefore, the threat by the $\mathrm{VC}$ can be circumvented by forgoing the ex ante control transfer in good state contracts. In this case, as seen above, truthful revelation is always guaranteed.

Second, renegotiation may be profitable for the E analogous to scenario 1. We know that $D_{g}=I$, i.e. the VC's claim is guaranteed independently of her effort level. In proof 1 , we showed that contracts are then renegotiation-proof for $D_{g} \geq C=I$ independently of $\lambda$. Thus to sum up, by choosing $D_{g}=I$ without control transfer, we obtain a renegotiationproof good-state contract which implements private first-best effort levels and achieves truth-revealing. 


\subsection{The Impact of Ex ante Grants on Contract Design}

$$
\text { 1. } A \leq[(1-p) q+(1-q) p]\left[I-l\left(a_{b}^{*}\right)\right]
$$

\begin{tabular}{|c|c|c|c|c|}
\hline Cases & Size of the spill-over effect & $E A G$ & C & $a_{b}$ \\
\hline $\begin{array}{c}1 \\
\text { (LLC) }\end{array}$ & $\begin{array}{c}S\left(e_{g}^{*}, a_{g}^{*}, \underline{\alpha}\right)>\frac{A}{p q}+ \\
{\left[1+\frac{(1-p)(1-q)}{p q}\right][I-l(0)]}\end{array}$ & $\begin{array}{c}S\left(e_{g}^{*}, a_{g}^{*}, \underline{\alpha}\right) \\
-\frac{(1-p)(1-q)}{p q}[I-l(0)]-\frac{A}{p q}\end{array}$ & $I$ & 0 \\
\hline $\begin{array}{c}2 \\
(\mathrm{LLC})\end{array}$ & $\begin{array}{c}{\left[I-l\left(a_{b}^{*}\right)\right]+\frac{(1-p)(1-q)}{p q}\left[I-L\left(a_{b}^{*}\right)\right]+\frac{A}{p q} \leq} \\
S\left(e_{g}^{*}, a_{g}^{*}, \underline{\alpha}\right)< \\
{\left[1+\frac{(1-p)(1-q)}{p q}\right][I-l(0)]+\frac{A}{p q}}\end{array}$ & $\begin{array}{c}S\left(e_{g}^{*}, a_{g}^{*}, \underline{\alpha}\right) \\
-\frac{(1-p q)(1-p)(1-q)}{p q} \frac{1}{2} \gamma \hat{a}_{E A G}^{2} \\
-\frac{(1-p)(1-q)}{p q}\left[I-l\left(\hat{a}_{E A G}\right)\right]-\frac{A}{p q}\end{array}$ & $\begin{array}{c}I \\
+(1-p)(1-q) \frac{1}{2} \gamma \hat{a}_{E A G}^{2}\end{array}$ & $\hat{a}_{E A G}$ \\
\hline $\begin{array}{c}3 \mathrm{a} \\
(\mathrm{LLC})\end{array}$ & $\begin{array}{c}\frac{(1-p)(1-q)}{p q}\left[I-L\left(a_{b}^{*}\right)\right]+\frac{A}{p q} \frac{1-(1-p)(1-q)}{(1-p) q+(1-q) p} \leq \\
S\left(e_{g}^{*}, a_{g}^{*}, \underline{\alpha}\right)< \\
{\left[I-l\left(a_{b}^{*}\right)\right]+\frac{(1-p)(1-q)}{p q}\left[I-L\left(a_{b}^{*}\right)\right]+\frac{A}{p q}}\end{array}$ & $\begin{array}{c}{[1-(1-p)(1-q)] S\left(e_{g}^{*}, a_{g}^{*}, \underline{\alpha}\right)-} \\
\frac{[(1-p) q+(1-q) p](1-p)(1-q)}{p q}\left[I-L\left(a_{b}^{*}\right)\right] \\
-\frac{A[1-(1-p)(1-q)]}{p q}\end{array}$ & $\begin{array}{c}I- \\
(1-p)(1-q) S\left(e_{g}^{*}, a_{g}^{*}, \underline{\alpha}\right) \\
+\frac{(1-p)(1-q)}{1-(1-p)(1-q)}\left[I-L\left(a_{b}^{*}\right)\right] \\
\left.\frac{p q+(1-p)(1-q)[(1-p) q+(1-q) p]}{p q}\right] \\
+\frac{(1-p)(1-q) A}{p q}\end{array}$ & $a_{b}^{*}$ \\
\hline $\begin{array}{c}3 \mathrm{a} \\
(\mathrm{PC})\end{array}$ & $\begin{array}{c}\frac{(1-p)(1-q)}{p q}\left[I-L\left(a_{b}^{*}\right)\right] \leq \\
S\left(e_{g}^{*}, a_{g}^{*}, \underline{\alpha}\right)< \\
\frac{(1-p)(1-q)}{p q}\left[I-L\left(a_{b}^{*}\right)\right]+\frac{A}{p q} \frac{1-(1-p)(1-q)}{(1-p) q+(1-q) p}\end{array}$ & $p q S\left(e_{g}^{*}, a_{g}^{*}, \underline{\alpha}\right)$ & $\begin{array}{c}I+\frac{1}{1-(1-p)(1-q)} \\
{\left[(1-p)(1-q)\left[I-L\left(a_{b}^{*}\right)\right]-\right.} \\
\left.p q(1-p)(1-q) S\left(e_{g}^{*}, a_{g}^{*}, \underline{\alpha}\right)\right]\end{array}$ & $a_{b}^{*}$ \\
\hline $\begin{array}{c}3 \mathrm{~b} \\
(\mathrm{PC})\end{array}$ & $\begin{array}{c}S\left(e_{g}^{*}, a_{g}^{*}, \underline{\alpha}\right)< \\
\frac{(1-p)(1-q)}{p q}\left[I-L\left(a_{b}^{*}\right)\right]\end{array}$ & $p q S\left(e_{g}^{*}, a_{g}^{*}, \underline{\alpha}\right)$ & $\begin{array}{c}I+\frac{(1-p)(1-q)}{p q} \\
{\left[I-L\left(a_{b}^{*}\right)\right]} \\
-(1-p q) S\left(e_{g}^{*}, a_{g}^{*}, \underline{\alpha}\right) \\
\end{array}$ & $a_{b}^{*}$ \\
\hline
\end{tabular}


2. $A=[(1-p) q+(1-q) p]\left[I-l\left(\tilde{a}_{b}\right)\right]$ mit $\tilde{a}_{b} \in\left(0, a^{*}-b\right)$

\begin{tabular}{|c|c|c|c|c|}
\hline Cases & Size of the spill-over effect & $E A G$ & $C$ & $a_{b}$ \\
\hline $\begin{array}{c}1 \\
(\mathrm{LLC})\end{array}$ & $\begin{array}{c}S\left(e_{g}^{*}, a_{g}^{*}, \underline{\alpha}\right)>\frac{A}{p q}+ \\
{\left[1+\frac{(1-p)(1-q)}{p q}\right][I-l(0)]}\end{array}$ & $\begin{array}{c}S\left(e_{g}^{*}, a_{g}^{*}, \underline{\alpha}\right) \\
-\frac{(1-p)(1-q)}{p q}[I-l(0)]-\frac{A}{p q}\end{array}$ & $I$ & 0 \\
\hline $\begin{array}{c}2 \\
(\mathrm{LLC})\end{array}$ & $\begin{array}{c}{\left[I-l\left(\tilde{a}_{b}\right)\right]+\frac{(1-p)(1-q)}{p q}\left[I-L\left(\tilde{a}_{b}\right)\right]+\frac{A}{p q} \leq} \\
S\left(e_{g}^{*}, a_{g}^{*}, \underline{\alpha}\right)< \\
{\left[1+\frac{(1-p)(1-q)}{p q}\right][I-l(0)]+\frac{A}{p q}}\end{array}$ & $\begin{array}{c}S\left(e_{g}^{*}, a_{g}^{*}, \underline{\alpha}\right) \\
-\frac{(1-p q)(1-p)(1-q)}{p q} \frac{1}{2} \gamma \hat{a}_{E A G}^{2} \\
-\frac{(1-p)(1-q)}{p q}\left[I-l\left(\hat{a}_{E A G}\right)\right]-\frac{A}{p q}\end{array}$ & $\begin{array}{c}I \\
+(1-p)(1-q) \frac{1}{2} \gamma \hat{a}_{E A G}^{2}\end{array}$ & $\hat{a}_{E A G}$ \\
\hline $\begin{array}{c}2 \\
(\mathrm{PC})\end{array}$ & $\begin{array}{c}\frac{1}{p q}\left[I-l\left(a_{b}^{*}\right)\right]+\frac{(1-p)(1-q)}{p q} \frac{1}{2} \gamma a_{b}^{* 2} \leq \\
S\left(e_{g}^{*}, a_{g}^{*}, \underline{\alpha}\right)< \\
\left.\left[I-l\left(\tilde{a}_{b}\right)\right]+\frac{(1-p)(1-q)}{p q}\left[I-L\left(\tilde{a}_{b}\right)\right)\right]+\frac{A}{p q}\end{array}$ & $\begin{array}{c}\frac{p q}{1-(1-p)(1-q)} S\left(e_{g}^{*}, a_{g}^{*}, \underline{\alpha}\right) \\
-\frac{[(1-p)(1-q)]^{2}}{1-(1-p)(1-q)} \frac{1}{2} \gamma \hat{a}_{E A G}^{2} \\
-\frac{(1-p)(1-q)}{1-(1-p)(1-q)}\left[I-l\left(\hat{a}_{E A G}\right)\right]\end{array}$ & $\begin{array}{c}I \\
+(1-p)(1-q) \frac{1}{2} \gamma \hat{a}_{E A G}^{2}\end{array}$ & $\hat{a}_{E A G}$ \\
\hline $\begin{array}{c}3 \mathrm{a} \\
(\mathrm{PC})\end{array}$ & $\begin{array}{c}\frac{(1-p)(1-q)}{p q}\left[I-L\left(a_{b}^{*}\right)\right] \leq \\
S\left(e_{g}^{*}, a_{g}^{*}, \underline{\alpha}\right)< \\
\frac{1}{p q}\left[I-l\left(a_{b}^{*}\right)\right]+\frac{(1-p)(1-q)}{p q} \frac{1}{2} \gamma a_{b}^{* 2}\end{array}$ & $p q S\left(e_{g}^{*}, a_{g}^{*}, \underline{\alpha}\right)$ & $\begin{array}{c}I+\frac{1}{1-(1-p)(1-q)} \\
{\left[(1-p)(1-q)\left[I-L\left(a_{b}^{*}\right)\right]-\right.} \\
\left.p q(1-p)(1-q) S\left(e_{g}^{*}, a_{g}^{*}, \underline{\alpha}\right)\right]\end{array}$ & $a_{b}^{*}$ \\
\hline $\begin{array}{c}3 \mathrm{~b} \\
(\mathrm{PC})\end{array}$ & $\begin{array}{c}S\left(e_{g}^{*}, a_{g}^{*}, \underline{\alpha}\right)< \\
\frac{(1-p)(1-q)}{p q}\left[I-L\left(a_{b}^{*}\right)\right]\end{array}$ & $p q S\left(e_{g}^{*}, a_{g}^{*}, \underline{\alpha}\right)$ & $\begin{array}{c}I+\frac{(1-p)(1-q)}{p q} \\
{\left[I-L\left(a_{b}^{*}\right)\right]-(1-p q) S\left(e_{g}^{*}, a_{g}^{*}, \underline{\alpha}\right)}\end{array}$ & $a_{b}^{*}$ \\
\hline
\end{tabular}

3. $A \geq[(1-p) q+(1-q) p][I-l(0)]$

\begin{tabular}{|c|c|c|c|c|}
\hline Cases & Size of the spill-over effect & $E A G$ & C & $a_{b}$ \\
\hline $\begin{array}{c}1 \\
\text { (LLC) }\end{array}$ & $\begin{array}{c}S\left(e_{g}^{*}, a_{g}^{*}, \underline{\alpha}\right)>\frac{A}{p q(1-p q)}+ \\
{\left[\frac{(1-p)(1-q)}{p q(1-p q)}\right][I-l(0)]}\end{array}$ & $\begin{array}{c}S\left(e_{g}^{*}, a_{g}^{*}, \underline{\alpha}\right) \\
-\frac{(1-p)(1-q)}{p q}[I-l(0)]-\frac{A}{p q}\end{array}$ & $I$ & 0 \\
\hline $\begin{array}{c}1 \\
(\mathrm{PC})\end{array}$ & $\begin{array}{c}\frac{1}{p q}[I-l(0)] \leq \\
S\left(e_{g}^{*}, a_{g}^{*}, \underline{\alpha}\right)<\frac{A}{p q(1-p q)}+ \\
{\left[\frac{(1-p)(1-q)}{p q(1-p q)}\right][I-l(0)]}\end{array}$ & $p q S\left(e_{g}^{*}, a_{g}^{*}, \underline{\alpha}\right)$ & $I$ & 0 \\
\hline $\begin{array}{c}2 \\
(\mathrm{PC})\end{array}$ & $\begin{array}{c}\frac{1}{p q}\left[I-l\left(a_{b}^{*}\right)\right]+\frac{(1-p)(1-q)}{p q} \frac{1}{2} \gamma a_{b}^{* 2} \leq \\
S\left(e_{g}^{*}, a_{g}^{*}, \underline{\alpha}\right)< \\
\frac{1}{p q}[I-l(0)]\end{array}$ & $p q S\left(e_{g}^{*}, a_{g}^{*}, \underline{\alpha}\right)$ & $\begin{array}{c}I \\
+(1-p)(1-q) \frac{1}{2} \gamma \hat{a}_{E A G}^{2}\end{array}$ & $\hat{a}_{E A G}$ \\
\hline $\begin{array}{c}3 \mathrm{a} \\
(\mathrm{PC})\end{array}$ & $\begin{array}{c}\frac{(1-p)(1-q)}{p q}\left[I-L\left(a_{b}^{*}\right)\right] \leq \\
S\left(e_{g}^{*}, a_{g}^{*}, \underline{\alpha}\right)< \\
\frac{1}{p q}\left[I-l\left(a_{b}^{*}\right)\right]+\frac{(1-p)(1-q)}{p q} \frac{1}{2} \gamma a_{b}^{* 2}\end{array}$ & $p q S\left(e_{g}^{*}, a_{g}^{*}, \underline{\alpha}\right)$ & $\begin{array}{c}I+\frac{1}{1-(1-p)(1-q)} \\
{\left[(1-p)(1-q)\left[I-L\left(a_{b}^{*}\right)\right]-\right.} \\
\left.p q(1-p)(1-q) S\left(e_{g}^{*}, a_{g}^{*}, \underline{\alpha}\right)\right]\end{array}$ & $a_{b}^{*}$ \\
\hline $\begin{array}{c}3 \mathrm{~b} \\
(\mathrm{PC})\end{array}$ & $\begin{array}{c}S\left(e_{g}^{*}, a_{g}^{*}, \underline{\alpha}\right)< \\
\frac{(1-p)(1-q)}{p q}\left[I-L\left(a_{b}^{*}\right)\right]\end{array}$ & $p q S\left(e_{g}^{*}, a_{g}^{*}, \underline{\alpha}\right)$ & $\begin{array}{c}I+\frac{(1-p)(1-q)}{p q} \\
{\left[I-L\left(a_{b}^{*}\right)\right]-(1-p q) S\left(e_{g}^{*}, a_{g}^{*}, \underline{\alpha}\right)}\end{array}$ & $a_{b}^{*}$ \\
\hline
\end{tabular}




\section{CFS Working Paper Series:}

\begin{tabular}{ll} 
No. & Author(s) \\
\hline $2006 / 28$ & $\begin{array}{l}\text { Christian Gollier } \\
\text { Alexander Muermann }\end{array}$
\end{tabular}

$2006 / 27$

\author{
Christian Laux \\ Uwe Walz
}

2006/26 Christian Laux

Alexander Muermann

$\begin{array}{ll}\text { 2006/25 } & \text { Bea Canto } \\ & \text { Roman Kräussl }\end{array}$

$2006 / 24$

\section{Toker Doganoglu \\ Christoph Hartz \\ Stefan Mittnik}

2006/23 Christoph Hartz

Stefan Mittnik

Marc S. Paolella

2006/22 Dirk Krueger

Hanno Lustig

Fabrizio Perri

2006/21 Juan Carlos Conesa

Sagiri Kitao

Dirk Krueger

2006/20 Annamaria Lusardi

Olivia S. Mitchell

2006/19 Carol C. Bertaut

Michael Haliassos

2006/18Ｄirk Krueger

Alexander Ludwig
Title

Optimal Choice and Beliefs with Ex Ante Savoring and Ex Post Disappointment

Tying Lending and Underwriting: Scope

Economies, Incentives, and Reputation

Mutual versus Stock Insurers: Fair Premium, Capital, and Solvency

Stock Market Interactions and the Impact of Macroeconomic News - Evidence from High

Frequency Data of European Futures Markets

Portfolio Optimization when Risk Factors are

Conditionally Varying and Heavy Tailed

Accurate Value-at-Risk Forecasting Based on the (good old) Normal-GARCH Model

Evaluating Asset Pricing Models with Limited

Commitment using Household Consumption Data

Taxing Capital? Not a Bad Idea After All!

Baby Boomer Retirement Security: The Roles of Planning, Financial Literacy, and Housing Wealth

Credit Cards: Facts and Theories

On the Consequences of Demographic Change for Rates of Returns to Capital, and the Distribution of Wealth and Welfare

Copies of working papers can be downloaded at http://www.ifk-cfs.de 